Virginia Commonwealth University

vCU Scholars Compass

2014

\title{
Electronic structure and magnetic properties of $\mathrm{Mn}$ and Fe impurities near the GaAs (110) surface
}

\author{
M. R. Mahani \\ Linnaeus University \\ M. Fhokrul Islam \\ Virginia Commonwealth University \\ A. Pertsova \\ Linnaeus University \\ C. M. Canali \\ Linnaeus University
}

Follow this and additional works at: http://scholarscompass.vcu.edu/phys_pubs

Part of the Physics Commons

Mahani, M.R., Islam, M.F., \& Pertsova, A., et al. Electronic structure and magnetic properties of Mn and Fe impurities near the GaAs (110) surface. Physical Review B, 89, 165408 (2014). Copyright (C 2014 American Physical Society.

\section{Downloaded from}

http://scholarscompass.vcu.edu/phys_pubs/33

This Article is brought to you for free and open access by the Dept. of Physics at VCU Scholars Compass. It has been accepted for inclusion in Physics Publications by an authorized administrator of VCU Scholars Compass. For more information, please contact libcompass@vcu.edu. 


\title{
Electronic structure and magnetic properties of Mn and Fe impurities near the GaAs (110) surface
}

\author{
M. R. Mahani, ${ }^{1}$ M. Fhokrul Islam, ${ }^{2}$ A. Pertsova, ${ }^{1}$ and C. M. Canali ${ }^{1}$ \\ ${ }^{1}$ Department of Physics and Electrical Engineering, Linnaeus University, 39182 Kalmar, Sweden \\ ${ }^{2}$ Virginia Commonwealth University, Richmond, Virginia 23284, USA \\ (Received 3 January 2014; revised manuscript received 28 March 2014; published 8 April 2014)
}

\begin{abstract}
Combining density functional theory calculations and microscopic tight-binding models, we investigate theoretically the electronic and magnetic properties of individual substitutional transition-metal impurities (Mn and $\mathrm{Fe}$ ) positioned in the vicinity of the (110) surface of GaAs. For the case of the $\left[\mathrm{Mn}^{2+}\right]^{0}$ plus acceptor-hole $(h)$ complex, the results of a tight-binding model including explicitly the impurity $d$ electrons are in good agreement with approaches that treat the spin of the impurity as an effective classical vector. For the case of Fe, where both the neutral isoelectronic $\left[\mathrm{Fe}^{3+}\right]^{0}$ and the ionized $\left[\mathrm{Fe}^{2+}\right]^{-}$states are relevant to address scanning tunneling microscopy (STM) experiments, the inclusion of $d$ orbitals is essential. We find that the in-gap electronic structure of Fe impurities is significantly modified by surface effects. For the neutral acceptor state $\left[\mathrm{Fe}^{2+}, h\right]^{0}$, the magnetic-anisotropy dependence on the impurity sublayer resembles the case of $\left[\mathrm{Mn}^{2+}, h\right]^{0}$. In contrast, for $\left[\mathrm{Fe}^{3+}\right]^{0}$ electronic configuration the magnetic anisotropy behaves differently and it is considerably smaller. For this state we predict that it is possible to manipulate the Fe moment, e.g., by an external magnetic field, with detectable consequences in the local density of states probed by STM.
\end{abstract}

DOI: 10.1103/PhysRevB.89.165408

PACS number(s): 75.50.Pp, 71.55.Eq, 71.15.Mb

\section{INTRODUCTION}

The study of the spin of individual transition-metal (TM) dopants in a semiconductor host is an emergent field known as magnetic solotronics, bearing exciting prospects for novel spintronics devices at the atomic scale [1]. The development of scanning tunneling microscopy (STM) based techniques enabled the investigation of substitutional dopants at a semiconductor surface with unprecedented accuracy and degree of details [2-9]. The experimental advances have stimulated theoretical studies of individual magnetic impurities in semiconductors, based both on first-principles calculations [10-15] and microscopic tight-binding (TB) models [16-24]. Approaches based on the TB models are particularly convenient to explore the solotronics limit of dilute impurities in semiconductor hosts. For the specific case of Mn dopants on the (110) GaAs surface, TB models [16,17,19-22,24] have provided a quantitative description of the electronic and magnetic properties of the associated acceptor states.

Early work by Tang and Flatté [16] introduced a TB model for a single substitutional Mn impurity in bulk GaAs. Here the electronic structure of the host is described by an $s p$ TB Hamiltonian, while the hybridization between the Mn $d$ levels and the $p$ levels of the nearest-neighbor As atoms is modeled as an effective spin-dependent potential. This model captures several of the key features of the Mn acceptor physics in GaAs found in experiment, such as the anisotropic shape of the Mn acceptor wave function. However, the inclusion of surface effects are expected to be important for a direct comparison with STM experiments, where accessible impurities are positioned in the proximity of the surface.

Later experimental [25] and theoretical [19,20] studies have indeed demonstrated a strong influence of the surface on the properties of the Mn acceptor state. In particular, the electronic structure and magnetic properties of Mn on the (110) GaAs surface and in near-surface layers have been investigated theoretically in Ref. [20]. This work uses a finite-cluster $s p^{3}$ TB model for GaAs, with the Mn impurity spin introduced as a classical spin, exchange coupled to the quantum spins of the nearest-neighbor As atoms. For a substitutional Mn in bulk GaAs this model is in good agreement with Ref. [16]. For a $\mathrm{Mn}$ on the surface the model finds, in agreement with experiment [4,6], a strongly localized and anisotropic midgap acceptor state. Furthermore, the model makes predictions on the dependence of the acceptor binding energy and magnetic anisotropy energy on the subsurface layer where the Mn is positioned. The former result has been later confirmed experimentally [7].

The theoretical approach used both in Ref. [16] and Ref. [20] must be viewed as an effective spin model for the Mn spin, where the Mn $d$ levels have been integrated out and the Mn spin is treated as a classical vector. The fact that this effective model makes predictions consistent with experiment, and that it also agrees with TB approaches retaining a microscopic description of $d$ levels [19], is a strong indication that the effective classical-spin approach is essentially correct for a $\mathrm{Mn}$ impurity in GaAs, characterized by a half-filled $d$ shell. The model does have some limitations, which are recognized and understood [24]. Other TM impurities in GaAs are less investigated and understood, and more refined microscopic theories are necessary. This is one of the motivations of the present work. The need of a microscopic description of TM impurities on a semiconductor surface is also motivated by recent STM experimental studies of Fe dopants on the (110) surface of GaAs [26-28]. Despite similarities in the STM topographies of Mn and Fe impurities as a result of the underlying $T_{2}$ symmetry of the host material [26], the electronic structures of $\mathrm{Mn}$ and $\mathrm{Fe}$ ions within the GaAs gap are known to be different than Mn [29], and this should have consequences in the STM spectroscopic features. This can already be seen in Fig. 1, where we draw schematically possible electronic configurations of $\mathrm{Mn}$ and $\mathrm{Fe}$ ions in bulk GaAs. In fact, two experimental groups have reported somewhat different results in the details of the Fe-impurity-induced in-gap electronic structure probed by STM. Richardella et al. [26] found two impurity-induced peaks 


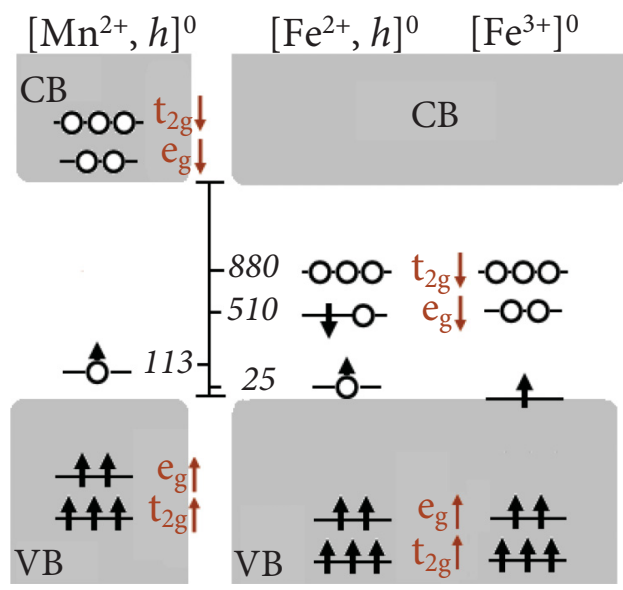

FIG. 1. (Color online) Transition-metal impurity levels in bulk GaAs host. The hybridization between Mn $3 d$ and nearest-neighbor As $3 p$ electrons gives rise to a bound hole at $113 \mathrm{meV}$ above the valence band while this weakly bound hole for the case of $\left[\mathrm{Fe}^{2+}, h\right]^{0}$ is at $25 \mathrm{meV}$. In the $\left[\mathrm{Fe}^{3+}\right]^{0}$ state, the electron is filling the weakly bound hole and contributing to the binding.

in their spectroscopic measurements, while a more recent study reported several peaks below and above the valence band maximum, which can be associated with the Fe impurity [28].

Another aspect of the complex electronic structure of Fe dopants on the (110) surface of GaAs has been investigated in the STM experiments by Bocquel et al. [27]. The authors demonstrated the possibility of manipulating the $d$-shell occupancy of Fe via a voltage-dependent local manipulation of the Fermi level due to tip-induced band bending. In particular, a transition from an isoelectronic $\left[\mathrm{Fe}^{3+}\right]^{0}$ to an ionized acceptor $\left[\mathrm{Fe}^{2+}\right]^{-}$state (see Fig. 1) was realized by varying the tip voltage. This transition corresponds to the change in the filling of the impurity $d$ shell from five $\left(d^{5}\right)$ to six $\left(d^{6}\right)$ electrons and therefore to the change in the core spin state from $S=5 / 2$ to $S=2$. It is clear that the classical-spin model, used to described the neutral $\left[\mathrm{Mn}^{2+}\right]^{0}$ plus acceptor-hole $(h)$ complex $\left(\left[\mathrm{Mn}^{2+}, h\right]^{0}\right)$, is not applicable in this case, as it cannot account for the change in the valence state of $\mathrm{Fe}$.

In this paper we employ a combined approach using both density functional theory (DFT) calculations and microscopic TB modeling to study the electronic structure and magnetic properties of substitutional $\mathrm{Mn}$ and $\mathrm{Fe}$ impurities on the (110) GaAs surface and up to nine monolayers below the surface. In our TB model we include explicitly the $s, p$, and $d$ orbitals of the impurity atom. The relative shifts in the on-site energies for the exchange-split minority and majority $d$ levels of the dopant are extracted from DFT calculations. For the case of Mn we find good agreement with previous work, where the Mn magnetic moment was treated classically. This indicates that our microscopic TB model correctly reproduces the physics of the $\left[\mathrm{Mn}^{2+}, h\right]^{0}$ complex, while providing access to additional features inaccessible by the classical spin model.

In the case of $\mathrm{Fe}$, we perform DFT calculations for both bulk and (110) GaAs surface. Here our DFT calculations provide a very valuable input for understanding the changes in the in-gap electronic states due to the proximity to the surface, and for interpreting the results of recent STM experiments.
Furthermore, using the TB model combined with parameters extracted directly from DFT calculations, we provide a detailed analysis of the relevant electronic and magnetic properties of $\mathrm{Fe}$ on the (110) GaAs surface. In particular, we calculate the spatial distribution of the wave functions associated with the impurity-induced states in the gap as well as the magnetic anisotropy and its dependence on the valence state of the impurity. We show that for the neutral acceptor state $\left[\mathrm{Fe}^{2+}, h\right]^{0}$, the magnetic-anisotropy dependence on the impurity position with respect to the surface layer is similar to that of $\left[\mathrm{Mn}^{2+}, h\right]^{0}$. In contrast to this, the isoelectronic state, $\left[\mathrm{Fe}^{3+}\right]^{0}$, behaves differently and its anisotropy energy is considerably smaller. This result suggests a route towards manipulation of the $\mathrm{Fe}$ magnetic moment by external magnetic fields, accompanied by detectable changes in the electronic local density of states (LDOS) which can be measured by STM. This is different from the case of Mn, where such manipulation was shown to be difficult since the large magnetic anisotropy energy (up to $15 \mathrm{meV}$ ) of $\mathrm{Mn}$ in the subsurface layers would require extremely strong magnetic fields to change the direction of its magnetic moment [24].

The rest of the paper is organized as follows. In Sec. II we describe the details of our microscopic TB approach. In Sec. III we present the results of DFT calculations for Mn and $\mathrm{Fe}$ impurities in GaAs, and discuss how these results can be used to extract some of the parameters of the TB Hamiltonian. The results of the TB calculations are described in Sec. IV. First, in Sec. IV A we present the calculations of the electronic energy spectrum, LDOS, and magnetic anisotropy for the Mn acceptor on the (110) GaAs surface and a few layers below it. In particular, we provide a quantitative comparison between the results obtained with our fully microscopic TB model and the results of the classical spin model for Mn, reported previously [20]. Second, in Sec. IV B we address similar properties of $\mathrm{Fe}$ dopants both in bulk GaAs and on the (110) GaAs surface. We focus in particular on the differences in the in-gap electronic structure, LDOS, and magnetic anisotropy associated with the Fe impurity in the bulk and on the surface. For the case of $\mathrm{Fe}$ on the surface, we show the dependence of the magnetic anisotropy on the valence states of the impurity and discuss possible implications on STM experiments. Finally, we draw some conclusions.

\section{MICROSCOPIC TIGHT-BINDING MODEL}

We use a multiorbital TB model to describe TM impurities substituting $\mathrm{Ga}$ atoms in GaAs. The model includes $s$ and $p$ orbitals for all Ga and As atoms and $s, p$, and $d$ orbitals for the impurity atoms. Introducing the $d$ orbitals for the impurities only, and not for Ga atoms, is justified by the fact that, as we will show in the next section, the $d$ levels of Ga are located far below $(\approx 15 \mathrm{eV})$ the Fermi level (see Fig. 4).

The second-quantized TB Hamiltonian for (Ga, TM)As takes the following form:

$$
H=H_{\mathrm{GaAs}}+H_{\mathrm{TM}}+H_{\mathrm{LRC}} .
$$

The first term in Eq. (1) is the TB Hamiltonian for the GaAs host (with the exclusion of the Ga atoms replaced by the TM 
impurity). It is the sum of two terms

$$
H_{\mathrm{GaAs}}=H_{\mathrm{band}}+H_{\mathrm{SOI}} \text {, }
$$

where

$$
H_{\mathrm{band}}=\sum_{i j, \mu \mu^{\prime}, \sigma} t_{\mu \mu^{\prime}}^{i j} a_{i \mu \sigma}^{\dagger} a_{j \mu^{\prime} \sigma}
$$

is the usual TB-band Hamiltonian for bulk GaAs [30] written in terms of Slater-Koster parameters, $\left(t_{\mu \mu^{\prime}}^{i j}\right)[31,32]$, representing both on-site energies and nearest-neighbor hopping amplitudes. Here $a_{i \mu \sigma}^{\dagger}$ and $a_{i \mu \sigma}$ are electron creation and annihilation operators; $i$ and $j$ are atomic indices that run over all atoms other than the impurity, $\mu$ and $\mu^{\prime}$ are orbital indices, and $\sigma=\uparrow, \downarrow$ is a spin index defined with respect to an arbitrary quantization axis. The one-body term

$$
H_{\mathrm{SOI}}=\sum_{i, \mu \mu^{\prime}, \sigma \sigma^{\prime}} \lambda_{i}\left\langle\mu, \sigma|\vec{L} \cdot \vec{S}| \mu^{\prime}, \sigma^{\prime}\right\rangle a_{i \mu \sigma}^{\dagger} a_{i \mu^{\prime} \sigma^{\prime}}
$$

models the on-site spin-orbit interaction (SOI) in GaAs, with the values of the renormalized spin-orbit splittings $\lambda_{i}$ taken from Ref. [30]. The second term in Eq. (1) describes the TM impurity and its coupling to the atoms of the host. We have

$$
\begin{aligned}
H_{\mathrm{TM}}= & \sum_{i, m, \mu, \nu, \sigma}\left(t_{\mu \nu}^{i m} a_{i \mu \sigma}^{\dagger} a_{m \nu \sigma}+t_{\mu \nu}^{i m \star} a_{m \nu \sigma}^{\dagger} a_{i \mu \sigma}\right) \\
& +\sum_{m, v, \sigma} \epsilon_{m \nu \sigma} a_{m \nu \sigma}^{\dagger} a_{m \nu \sigma}+H_{\mathrm{SOI}}^{\mathrm{TM}}
\end{aligned}
$$

where $a_{m \nu \sigma}^{\dagger}$ and $a_{m v \sigma}$ are creation and annihilation operators at the impurity site $m$; the orbital index $v$ runs over $s, p$, and $d$ orbitals. The first term in Eq. (5) describes a hopping between the impurity and its As nearest neighbors. For the Slater-Koster hopping parameters between the impurity $d$ orbitals and the nearest-neighbor As $s$ and $p$ orbitals we use the same values as for the corresponding hopping parameters between $\mathrm{Ga}$ and As, which are available in the literature [33]. Our tight-binding model must be viewed as a phenomenological approach which allows us to introduce, in a physically meaningful way, the microscopic $d$ levels for the impurity and to go beyond the classical-spin model used previously.

The second term in Eq. (5) represents on-site energies of the impurity for a given orbital. The $d$-orbital energies $\epsilon_{m} d \sigma$ play an important role in the model. Their values for "spin-up" (majority) and "spin-down" (minority) electrons are different, which leads to a different occupation for opposite spin states, and hence to a nonzero spin magnetic moment at the impurity site. As a first estimate of the on-site $d$-orbital energies, we use the values of the exchange-split majority and minority $d$ levels, which can be identified in the spin- and orbital-resolved density of states (DOS) of the impurity, calculated with DFT. The exact procedure and the choice of the $d$-orbital on-site energies for specific cases of $\mathrm{Mn}$ and $\mathrm{Fe}$ in GaAs will be discussed in Sec. III. The last term in Eq. (5) is an on-site spin-orbit coupling term for the impurity atom, analogous to the one given in Eq. (4). The spin-orbit coupling terms $H_{\mathrm{SOI}}$ and $H_{\mathrm{SOI}}^{\mathrm{TM}}$ will cause the total ground-state energy of the system to depend on the direction of the impurity magnetic moment, defined with respect to an arbitrary quantization axis. This is the origin of the magnetic anisotropy energy.
Finally, the third term in Eq. (1),

$$
H_{\mathrm{LRC}}=\frac{e^{2}}{4 \pi \varepsilon_{0} \varepsilon_{r}} \sum_{m} \sum_{i \mu \sigma} \frac{a_{i \mu \sigma}^{\dagger} a_{i \mu \sigma}}{\left|\vec{r}_{i}-\vec{R}_{m}\right|},
$$

is a long-range repulsive Coulomb potential that is dielectrically screened by the host material (the index $m$ runs over all impurity atoms). This term prevents extra electrons from approaching the impurity atom too closely and therefore prevents it from charging. This contributes to localizing the acceptor hole around the impurity. The dielectric constant $\epsilon_{r}$ for the impurity on the GaAs surface is reduced from the bulk $\mathrm{GaAs}$ value in order to mimic a weaker dielectric screening at the surface (12 for bulk and 6 for the surface). This crude choice is qualitatively supported by experimental results [7].

As already mentioned in the introduction, the modeling of the TM impurity considered here [see Eq. (5)] differs from the approach of Ref. [20] in that the TM impurity $d$ orbitals are introduced explicitly. In Ref. [20] the $d$ orbitals are integrated out and their spin-dependent hybridization with the As $p$ orbitals is represented by the following effective spin Hamiltonian:

$$
H_{\text {class-spin }}=J_{p d} \sum_{m} \sum_{i[m]} \vec{S}_{i} \cdot \hat{\Omega}_{m} .
$$

Equation (7) describes the antiferromagnetic exchange coupling (with coupling constant $J_{p d}>0$ ) between the TM impurity spin $\hat{\Omega}_{m}$ (treated as a classical vector) and the nearest-neighbor As $p$ spins $\vec{S}_{i}=1 / 2 \sum_{\pi \sigma \sigma^{\prime}} a_{i \pi \sigma}^{\dagger} \vec{\tau}_{\sigma \sigma^{\prime}} a_{i \pi \sigma^{\prime}}$. Below we will refer to the model of Eq. (7) as the classical-spin model, in contrast to the present impurity model, given in Eq. (5), which will be denoted as the quantum d-level model.

The electronic structure of GaAs with a single substitutional Mn or Fe impurity atom is obtained by performing supercelltype calculations with a cubic cluster of 3200 atoms and periodic boundary conditions in either 2 or 3 dimensions, depending on whether we are studying the (110) surface or a bulklike system. The (110) surface of GaAs is attractive from both theoretical and experimental points of view due to the absence of large surface reconstruction. In order to remove artificial dangling-bond states that would otherwise appear in the band gap, we include relaxation of surface layer positions following a procedure put forward in Refs. [34] and [35]. For more details the reader is referred to Ref. [20]. We would like to note here that while the effects of strain induced by the (110) surface relaxation in GaAs are included in our study, following the prescription of Refs. [34] and [35] mentioned above, we do not take into account the modification in strain and relaxation caused by the presence of the magnetic impurity. A systematic study of surface-induced strain and strain from an embedded quantum dot on the symmetry of an acceptor is presented, respectively, in Refs. [36] and [37]. In Ref. [38] the authors present a model for the on-site matrix elements of the $s p^{3} d^{5} s^{*}$ TB Hamiltonian of a strained diamond or zinc-blende crystal. Finally, in a more recent paper [39], the author presents a method to include strain into the tight-binding Hamiltonian, which is suitable for calculations of the electronic and optical properties of semiconductor nanosystems embedded in a host crystal. These extensions of the TB model represent future 
challenges to further improve the description of the strain effects induced by surfaces and impurities in semiconductors.

\section{DFT CALCULATIONS AND EXTRACTION OF THE TB PARAMETERS}

The DFT calculations are performed using the fullpotential all-electron method with the basis consisting of linearized augmented plane waves combined with local orbitals (LAPW + lo), as implemented in the WIEN2K package [40]. We use the generalized gradient approximation (GGA) with Perdew-Burke-Ernzerhof (PBE) exchange correlation functional [41] as well as GGA $+U$, where the orbital-dependent $U$ parameter is used to capture electronic correlations in the core shell of the impurity. Because of the time-expensive nature of the plane wave method, we have used the SIESTA $a b$ initio package, which employs pseudopotentials and a numerical basis set [42], for relaxing the surfaces in our calculations. The relaxed coordinates are then used as input for WIEN2K calculations.

For bulk calculations, we consider a $2 \times 2 \times 2$ supercell containing a total of 64 atoms, with one $\mathrm{Ga}$ atom replaced by a TM impurity atom. We use 100 nonequivalent $k$ points in the Brillouin zone. For the surface calculations, a $4 \times 2$ surface supercell with six layers, each containing 16 atoms, is constructed by cleaving the bulk crystal along the [110] direction. A vacuum of 25 bohrs is added along the surface to avoid supercell interaction. All the surface calculations are performed by substituting one Ga atom from the surface layer by a TM impurity. Note that due to computational limitations caused by the large size of the supercell we use one $k$ point in the surface calculations. This choice of $k$-point sampling can be justified based on the fact that the Brillouin zone for the surface supercell is considerably smaller than that of the bulk [15].

The DFT calculations carried out in this work provide the spin-resolved DOS for the $d$ electrons of Mn and Fe impurities in the bulk and on the surface of GaAs. Importantly, the splitting between the majority and the minority $d$ levels of the impurities, calculated with DFT, determines the relative values of the on-site energies for the corresponding majority and minority $d$ orbitals in our TB model. Figure 2 shows

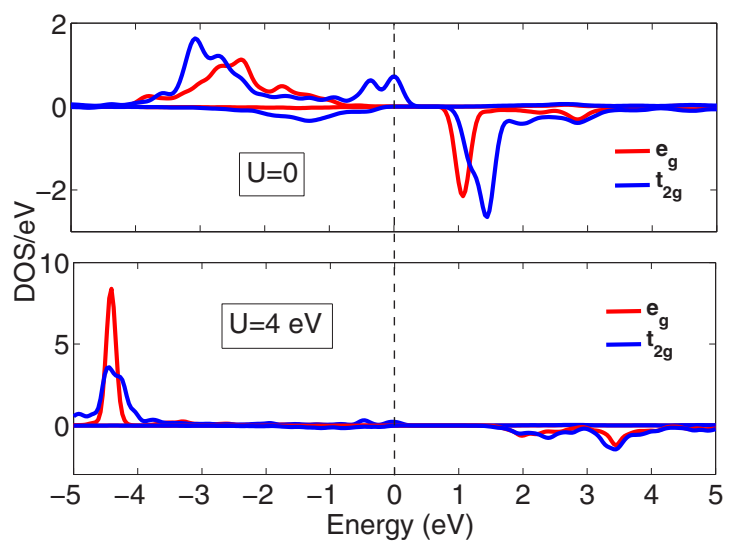

FIG. 2. (Color online) Spin-resolved DOS for the $d$ orbitals of the Mn dopant in bulk GaAs for $U=0$ (top panel) and $U=4 \mathrm{eV}$ (bottom panel). The vertical dashed line at $E=0$ denotes the position of the Fermi level. the spin-resolved DOS for the Mn dopant in bulk GaAs for two different choices of the $U$ parameter in the GGA $+U$ calculations. The $U$ parameter tends to localize the majority $d$-shell electrons and push them deeper into the valence band. It is clear from the figure that the separation between majority (spin-up) and minority (spin-down) $d$ orbitals of different symmetry $\left(e_{g}\right.$ and $\left.t_{2 g}\right)$ increases with increasing value of $U$.

A microscopic determination of the parameter $U$ in firstprinciples $\mathrm{GGA}+U$ (or $\mathrm{LDA}+U$ ) calculations in complex materials is an outstanding and complicated issue in computational physics and material science, details of which were first addressed in Ref. [43]. For the specific case of TM impurities in semiconductors, more information is available on the value of $U$ for $\mathrm{Mn}$ in GaAs via comparison with experiment and calculations, while for the case of $\mathrm{Fe}$ dopants, information is scarcer. We believe that neglecting $U$ altogether is not justified, since some kind of self-interaction correction to the TM must be included and it does have an effect on the electronic structure, noticeably the position of the $d$ levels. Our approach has been to choose a value not very different from the value of $U$ for $\mathrm{Mn}$, under the assumption that the two cases should be qualitatively similar and that small variations of $U$, if present, should not change the results substantially. References [44] and [45] both use $U=4 \mathrm{eV}$ in Fe-doped GaN semiconductors. In Ref. [46], which investigates several transition-metal-doped $\mathrm{ZnO}$ semiconductors with $\mathrm{LDA}+U$, the value of $U=4.5 \mathrm{eV}$ (and exchange $J=0.5 \mathrm{eV}$ ) is used for all transition metals. This paper mentions explicitly that although small variations are expected across the TM series, the choice of constant values permits a more straightforward comparison, which is precisely our point of view on this issue. The value of $U$ is usually chosen to match photoemission spectra and in our calculation we use $U=4 \mathrm{eV}$ [44], although the results for $U=0$ are also presented.

The values of the on-site TB parameters for the Mn $d$ orbitals in bulk are determined based on the calculations with $U=4 \mathrm{eV}$ (see the bottom panel of Fig. 2). As a first estimate for these parameters we take the positions of the spin-resolved $e_{g}$ and $t_{2 g}$ levels. We then tune the value of the on-site energy for the majority $t_{2 g}$ orbital to get the exact position of the acceptor level introduced by $\mathrm{Mn}$ in the bulk GaAs gap. The values of the on-site energies are summarized in Table I.

The spin-resolved DOS for Mn on the (110) GaAs surface is shown in Fig. 3. The lower symmetry on the surface lifts the degeneracy of the $d$-orbitals and, strictly speaking, we can no longer identify peaks corresponding to orbitals with $e_{g}$ and $t_{2 g}$ symmetry as in the bulk case. However, as one can see from the bottom panel of Fig. 3, in the case of $U=4 \mathrm{eV}$ the positions of the main peaks in the surface DOS are quantitatively similar to the positions of the $e_{g}$ and $t_{2 g}$ peaks in the bulk calculation

TABLE I. The on-site energies of $\mathrm{Mn}$ and $\mathrm{Fe} d$ orbitals in $\mathrm{eV}$.

\begin{tabular}{lcclc}
\hline \hline & Mn (bulk) & Mn (surface) & Fe (bulk) & Fe (surface) \\
\hline$e_{g}^{u p}$ & -4.5 & -4.5 & -6.5 & -4.5 \\
$t_{2 g}^{u p}$ & -2.226 & -2.226 & -6.5 & -4.5 \\
$e_{g}^{d n}$ & 3.5 & 3.5 & -0.138 & -1 \\
$t_{2 g}^{d n}$ & 3.5 & 3.5 & -0.02 & -1 \\
\hline \hline
\end{tabular}




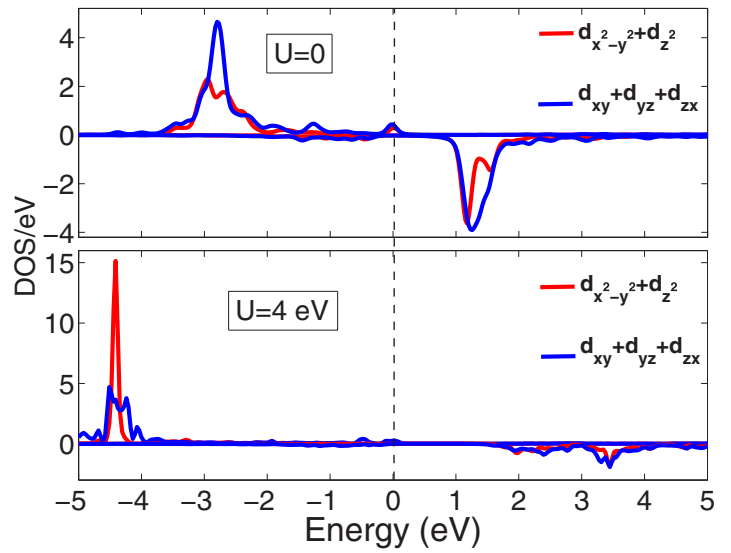

FIG. 3. (Color online) Spin-resolved DOS for the $d$ orbitals of the Mn dopant on the (110) GaAs surface for $U=0$ (top panel) and $U=4 \mathrm{eV}$ (bottom panel). The vertical dashed line at $E=0$ denotes the position of the Fermi energy.

with the same value of $U$ (bottom panel of Fig. 2). Therefore, in our TB calculations for Mn on the (110) GaAs surface we use the same set of parameters as for Mn in bulk. As we will show in Sec. IV A, the properties of the acceptor state obtained from these calculations are in good agreement with the STM experiments.

In Fig. 4 we also plot the spin-resolved density of states for the $d$ orbitals of a Ga atom on the (110) GaAs surface, and compare it with the analogous quantity for a substitutional Mn atom. We can clearly see that the Ga $d$ orbitals are, as expected, unpolarized and occur at energies far below the Fermi level. The band structure of bulk GaAs (see Fig. 5) reveals that the top of the valence band in GaAs is completely dominated by $p$-like states, both for $\mathrm{Ga}$ and As. Levels of $d$ character are visibly present, particularly for Ga atoms, even at the Gamma point. However, their contribution is only $2 \%$ of the $p$-like states. These results justify our simplifying choice of disregarding Ga $d$ orbitals in the TB model. We would like to emphasize that the $d$-level parameters in the empirical TB model can differ considerably from the corresponding electronic atomic orbitals. Therefore our remark on the position of the $a b$ initio Ga $d$-level energy being far away from the Fermi energy as a justification of our neglect of

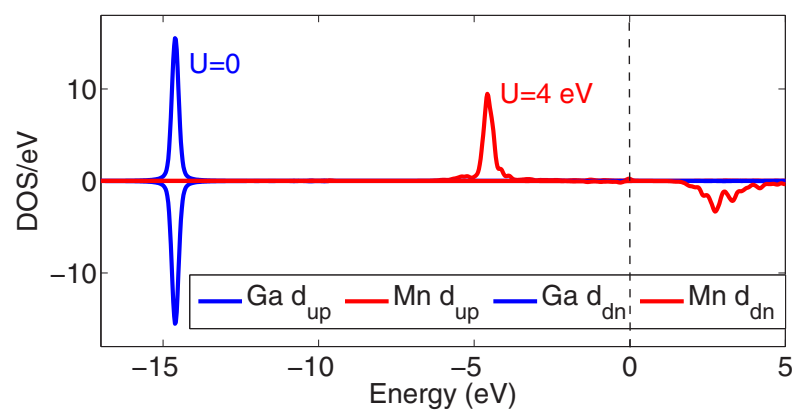

FIG. 4. (Color online) Spin-resolved density of states (DOS) for the $d$ orbitals of a Ga atom and that of a substitutional $\mathrm{Mn}$ on the (110) GaAs surface. The vertical dashed line at $E=0$ denotes the position of the Fermi level.

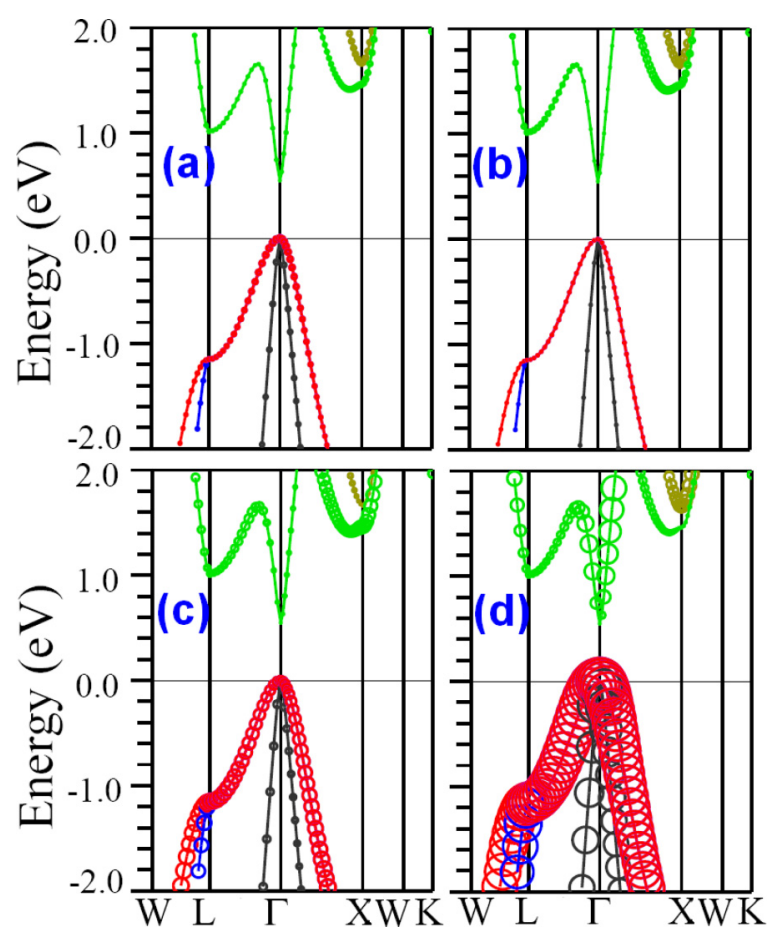

FIG. 5. (Color online) Band structure of bulk GaAs along the high-symmetry point in the Brillouin zone. (a)-(d) Band character of Ga $d$, As $d$, Ga $p$, and As $p$ orbitals respectively. The radius of the circles represents the corresponding weight for the particular $k$ and energy point. The horizontal black line at $E=0$ denotes the position of the Fermi level.

these levels in the TB model must be taken with caution. Note, however, that the parametrization of GaAs that we adopt here, which excludes $d$ levels of Ga and As atoms, is a standard procedure and therefore the $a b$ initio results are not inconsistent with neglecting the $\mathrm{Ga}$ and As $d$ levels in the TB model.

We will now discuss the case of Fe in GaAs. The spinresolved DOS for the Fe dopant in bulk GaAs is presented in Fig. 6. We note that our calculations with $U=0$ for both

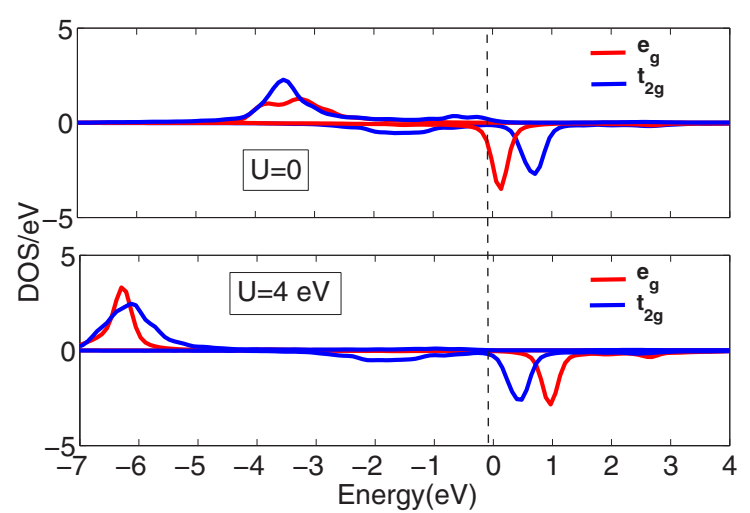

FIG. 6. (Color online) Spin-resolved DOS for the $d$ orbitals of the Fe dopant in bulk GaAs for $U=0$ (top panel) and $U=4 \mathrm{eV}$ (bottom panel). The vertical dashed line at $E=0$ denotes the position of the Fermi level. 


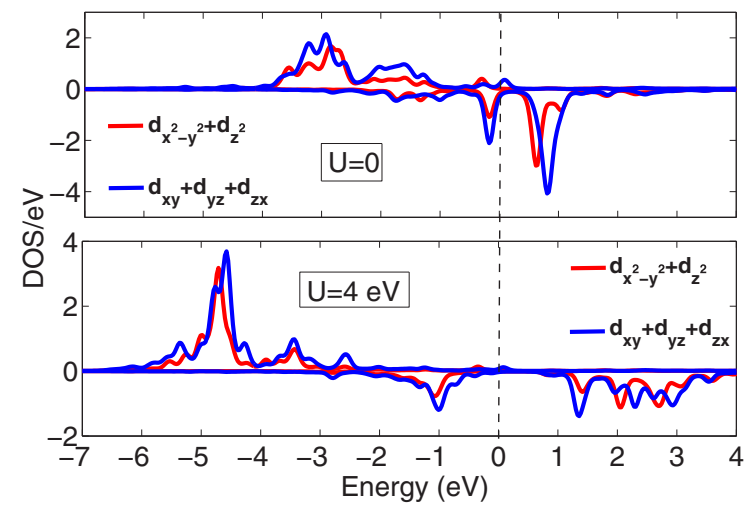

FIG. 7. (Color online) Spin-resolved DOS for the $d$ orbitals of the Fe dopant on the (110) GaAs surface for $U=0$ (top panel) and $U=4 \mathrm{eV}$ (bottom panel). The vertical dashed line at $E=0$ denotes the position of the Fermi level.

$\mathrm{Fe}$ and $\mathrm{Mn}$ in bulk GaAs are in agreement with previously reported results [11]. As one can see from Fig. 6, the majority $e_{g}$ and $t_{2 g}$ peaks are pushed deeper in the valence band for $U=4 \mathrm{eV}$ compared to the case with $U=0$. Interestingly, the positions of the minority $e_{g}$ and $t_{2 g}$ peaks above the Fermi level are sensitive to the value of $U$. The minority doublet $\left(e_{g}\right)$ level is lower in energy than the minority triplet $\left(t_{2 g}\right)$ level for the case with $U=0$; however these two levels swap for $U=4 \mathrm{eV}$. Based on the calculation with $U=4 \mathrm{eV}$, we choose the positions of the two main peaks for the majority $d$ levels $(-6.5 \mathrm{eV})$ as the on-site energy of the corresponding $d$ orbitals in our TB model for Fe in bulk. We then tune the value of the on-site energy for the minority $e_{g}$ and $t_{2 g}$ orbitals to get the exact electronic structure of $\mathrm{Fe}$ in bulk GaAs [29]. We tune these two parameters to avoid any complications caused by swapping of the two minority peaks for different $U$ parameters.

The results of the DFT calculations for the Fe dopant on the (110) surface of GaAs are shown in Fig. 7. The lower symmetry on the surface lifts the degeneracy of the $d$ orbitals and more peaks appear in the DOS compared to the bulk. The values of the TB parameters of the $d$ orbitals of $\mathrm{Fe}$ on the surface are again extracted directly from the DFT calculation with $U=4 \mathrm{eV}$ (see the bottom panel of Fig. 7) as the positions of the peaks in the spin-resolved DOS for the corresponding orbitals. In contrast to $\mathrm{Mn}$, we do not tune the extracted values for $\mathrm{Fe}$ on the GaAs surface. This is due to the lack of accepted experimental data on the position of the impurity-induced states in the gap for the case of $\mathrm{Fe}$ on the surface. We take the value for the spin-up on-site energy of $e_{g}$ and $t_{2 g}$ orbitals from the main peak of the majority DOS, which is located at $\approx 4.5 \mathrm{eV}$. In the case of the minority DOS, we find two pronounced peaks, one above and the other one below zero. The choice of the positive on-site energy for the spin-down $e_{g}$ and $t_{2 g}$ orbitals pushes the Fe levels into the conduction band, which seems to be an unlikely scenario based on the bulk level structure. Therefore we take the value for the on-site energy for spin-down $e_{g}$ and $t_{2 g}$ orbitals from the peak in the minority DOS located at $\approx-1 \mathrm{eV}$ (see Table I).

\section{RESULTS AND DISCUSSION}

\section{A. Mn dopants on (110) GaAs surface}

We start by discussing the results of TB modeling of a single Mn dopant in GaAs. As we will see in the following, our TB model incorporating the impurity $d$ orbitals reproduces the well-known features of $\mathrm{Mn}$ in the bulk and on the surface of $\mathrm{GaAs}$, in agreement with previous studies. However, we also find some interesting differences between our approach and the classical-spin model [20]. These differences are mainly related to the magnetic anisotropy of $\mathrm{Mn}$ on the surface and in the subsurface layers.

Figure 8 shows the electronic properties of $\mathrm{Mn}$ in the bulk (top panels) and on the (110) surface (bottom panels) of GaAs. $\mathrm{Mn}$ introduces three levels in the GaAs gap, with the highest level, which is unoccupied, known as the hole-acceptor level. The other two levels are occupied and they lie below the acceptor. The position of the acceptor level with respect to the valence band is found at $113 \mathrm{meV}$ for the bulk and at $0.89 \mathrm{eV}$ for the surface dopant. While the bulk calculation reproduces exactly the experimental value [47-50] (see the discussion about the TB parametrization in Sec. III), the surface calculation also gives the position of the acceptor level close to the experimental result [4]. As one can see from Fig. 8(c), the calculated LDOS for the acceptor on the surface shows more concentration of the spectral weight on the $\mathrm{Mn}$ site compared to the bulk case, which is an indication of a deeper and a more localized state. In general, the calculations presented in Fig. 8 support the results of the classical spin model $[16,20]$ and are in good agreement with other theoretical and experimental results $[4,19]$.

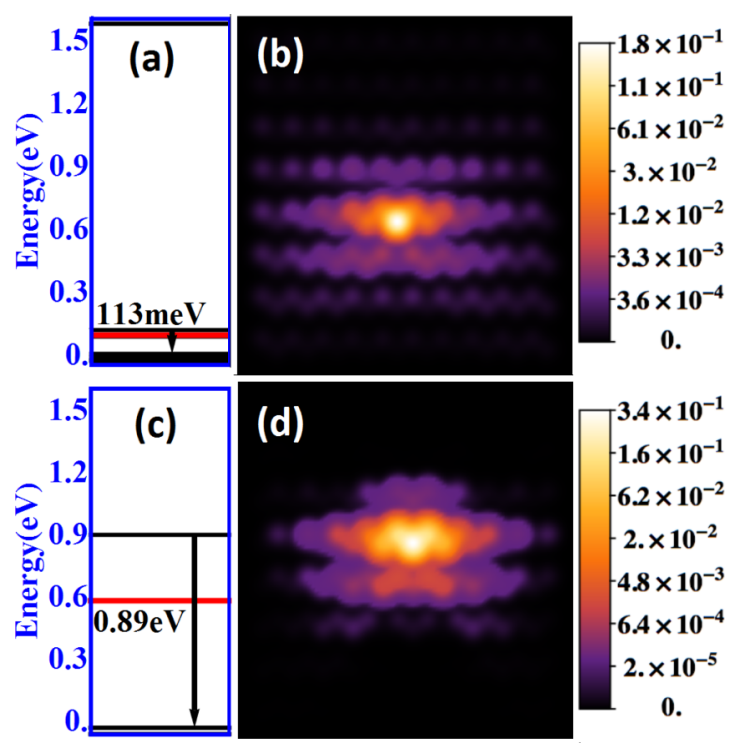

FIG. 8. (Color online) Electronic properties of $\mathrm{Mn}$ in GaAs, calculated using the TB model in which the Mn $d$ levels are included explicitly. Eigenvalue spectrum [(a), (c)] and the calculated LDOS for the acceptor state [(b), (d)]. Top panels are for Mn in bulk; bottom panels are for Mn on the surface. In panels (a) and (c) the red lines mark the highest occupied level while the black lines mark the acceptor state (the first level above the highest occupied level). 
We would like to comment on one important feature of the electronic structure of Mn in bulk GaAs. According to Fig. 8(a) the three levels introduced by $\mathrm{Mn}$ in the bulk GaAs gap are found to be spread over an energy interval of approximately $30 \mathrm{meV}$, when SOI is included in the calculations. (In the figure, the topmost level and the lowest level in the gap are split by $\approx 30 \mathrm{meV}$.) This is a shortcoming that the present quantum $d$-level model shares with the classical-spin models of Refs. [16,20]: In fact the three (predominantly) $p$ levels appearing in the gap should be degenerate in the perfectly tetragonal environment of an impurity in bulk GaAs. The lifting of the degeneracy is connected with the breaking of time reversal and rotational symmetry in mean-field-like treatments of the kinetic-exchange coupling between the TM impurity $d$ levels and the $p$ levels of the nearest-neighbor As atoms.

The symmetry of the Mn acceptor ground state in bulk GaAs is an important issue. The correct framework to discuss this problem is within a many-body approach. For a $\mathrm{Mn}$ in bulk GaAs, a simplified many-body Hamiltonian that captures the salient features of the problem consists of the Mn impurity (with ten $d$ levels occupied on average by five electrons) and the four nearest-neighbor As atoms. The problem is eventually reduced to a sum of three terms: the acceptor Hamiltonian (with single-particle degeneracy equal to six corresponding to twice the number of $p$ levels) which in the GS is spanned by states with five electrons (or, equivalently, one hole); the Mn-impurity part with (on average) five electrons localized in the $d$ orbitals of $\mathrm{Mn}^{2+}$; and a hopping Hamiltonian describing the $p$ - $d$ hybridization between As $p$ orbitals and $\mathrm{Mn}^{2+} d$ orbitals. This finite-cluster many-electron model captures the tetragonal symmetry of the system, although (being a finite system) it is only a rudimentary representation of the valence-band structure at the $\Gamma$ point.

The many-body Hamiltonian can be solved approximately by second-order perturbation theory in the hopping parameters. In the paramagnetic regime, which conserves timereversal symmetry, the model yields a threefold degenerate GS, corresponding to the lowest-energy spin-multiplet $J=1$, describing the effective exchange antiferromagnetic coupling between the hole spin $j=3 / 2$ and the manganese spin $S=$ $5 / 2$. The preservation of time-reversal symmetry, which can be enforced in this many-body approach, is crucial. Note that on the general basis of group theory, irreducible representations of $S=1$ in a tetragonal symmetry must necessarily be degenerate. The case considered here is an example of this general property. This property is lost when the Mn quantum spin is replaced by a classical spin vector pointing in some arbitrary direction. Likewise and for the same reason, it is also lost in any mean-field treatment of the many-body Hamiltonian describing the Mn impurity. This is the case of our quantum Hamiltonian, where the $d$ levels are chosen to be spin polarized in a similar arbitrary but fixed direction, effectively behaving like an external magnetic field, which breaks both time and rotational invariance. Note that this is also the case of $a b$ initio spin density functional theory, whose rationale is quite close to our $d$-level model. The ensuing effective one-body Hamiltonian displays in three in-gap single-particle acceptor states of predominantly $p$ character, which are typically split by an amount of the order of the spin-orbit coupling strength.
Thus the GS has degeneracy 1 instead of 3, as derived in the many-body approach.

It turns out that this drawback of the effective one-particle Hamiltonian (either with the classical spin-model or with the mean-field treatment of the $d$ levels) and its incorrect description of the GS degeneracy is uninfluential when it comes to describing the properties of the Mn acceptor state which can be probed by STM experiments, for example. The reason is that, when an electron is added to the system (via electron tunneling, for example), the inclusion of an interaction Hubbard $U$ implies that only a single state is accessible even in the many-body approach. In other words, for typical values of the Hubbard $U$ parameter, a second-bound state is not found for the impurity(see, for example, the discussion in Ref. [16]). This important remark justifies the use of the effective single-particle models considered above, and explains their remarkable success in reproducing the main features of the Mn acceptor wave functions probed in STM experiments.

A perfect threefold degenerate level is expected for the present model and for the models of Refs. [16,20] when SOI is switched off. Indeed, our calculations show that (i) the splitting between the three levels in the gap, as well as the relative position of the acceptor level with respect to the top of the valence band (113 meV), remains unchanged in very large clusters consisting of up to 30000 atoms, when SOI is included, and (ii) the small splitting still present in the supercell calculations with 3200 atoms without SOI is instead purely a finite-size effect, which vanishes when increasing the size of the supercell: the splitting reduces from $11.54 \mathrm{meV}$ for 3200 atoms to $0.62 \mathrm{meV}$ for 20000 atoms. That is, in the absence of SOI, the splitting is zero for this model.

Figure 9 shows the magnetic-anisotropy-energy landscape for a single $\mathrm{Mn}$ in the bulk and on the (110) GaAs surface. The magnetic-anisotropy-energy landscape is defined as the ground-state energy of the system plotted for different directions of the spin quantization axis, that is, as a function of the angles $\theta$ and $\phi$ (polar and azimuthal angles, which define the direction of the quantization axis). The coordinate system used for these plots has $\theta=0$ parallel to the [001] direction and $\left(\theta=\frac{\pi}{2}, \phi=\frac{\pi}{2}\right)$ parallel to [010] direction. We compare the results obtained with our TB model (top panels) with the classical-spin model, introduced in Ref. [20] (bottom panels). The magnetic anisotropy landscape is similar for the two models. In particular, for Mn in bulk the models feature two bistable easy axes, which are parallel to the [001] direction and are separated by a barrier in the (001) plane [see Figs. 9(a) and $9(\mathrm{c})$ ]. The fact that the shape of the magnetic anisotropy landscape does not change means that the symmetry properties that control this quantity are correctly represented by the classical-spin model, or at least, they are captured in the same way as the more microscopic model.

Although the overall shapes of magnetic anisotropy landscapes for Mn on the surface are in qualitative agreement [see Figs. 9(b) and 9(d)], the anisotropy energy is one order of magnitude smaller in the case of our quantum $d$-level model. A smaller value of the anisotropy energy is consistent with the picture of a localized acceptor level (Fig. 8). The set of TB parameters extracted from GGA $+U$ calculations for $\mathrm{Mn}$ on the surface gives a deeper and a more localized acceptor state, compared to the calculations done with the classical spin model 
(a)

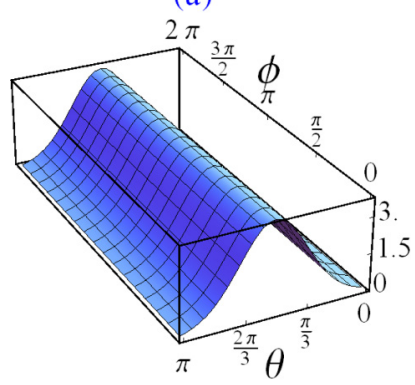

(c)

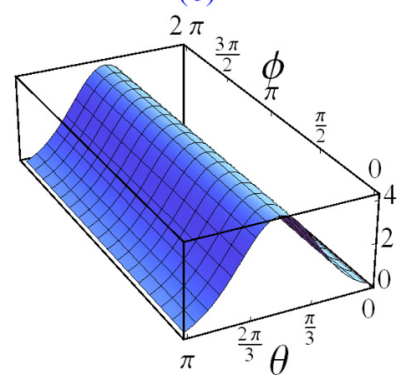

(b)

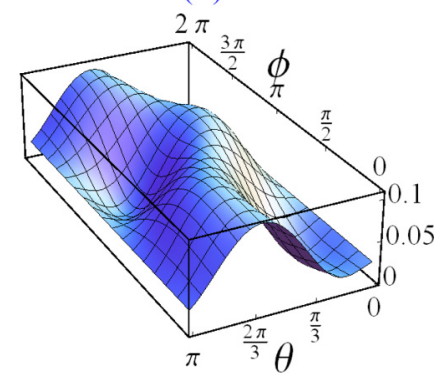

(d)

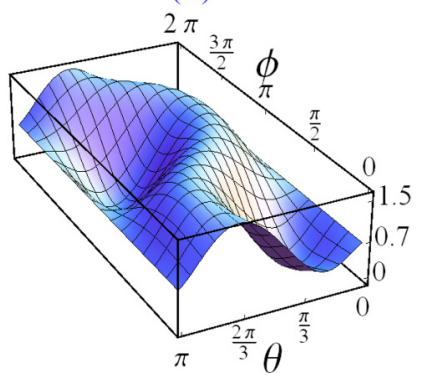

FIG. 9. (Color online) A comparison of magnetic anisotropy energy landscapes calculated with the fully microscopic model, which includes the Mn $d$ orbitals (top panels) and with the classical-spin model (bottom panels). Panels (a) and (c) are for bulk; (b) and (d) are for the surface. Energy is in the units of meV.

[20], which in turn leads to lower anisotropy. Figures 10 and 11 show the calculated LDOS and the anisotropy energy of the acceptor state when $\mathrm{Mn}$ is successively moved down from the surface layer towards the center of the cluster. Figure 11 shows a comparison of the anisotropy energy calculated with the two models as a function of the Mn depth, where we also include the values for the (110) surface and for the bulk. Here the value of the anisotropy energy is defined to be difference between the maximum and the minimum ground-state energy, calculated as a function of either the direction of magnetic moment in the case of classical-spin model, or the quantization axis in the quantum $d$-level model. We would like to point out several important features that can be seen on this figure. The lower value of the anisotropy energy compared to the classical-spin model, found in our calculations, persists for almost all of the considered subsurfaces. The relatively high anisotropy for the bulk found in both models is a finite-size effect. In fact, our calculations demonstrate that the bulk anisotropy energy drops to a small fraction of a meV when the number of atoms in the cluster is increased by a factor of 10 . As $\mathrm{Mn}$ is moved toward the center of the cluster, one expects the anisotropy energy to drop to its bulk value. The present cluster (3200 atoms) includes $20 \mathrm{Ga}$ layers along the [110] direction; therefore the ninth Ga sublayer is the last sublayer where we can replace a $\mathrm{Ga}$ with a Mn impurity atom. By increasing the size of the cluster we are able to place the Mn atom in the deeper sublayers. As expected, such calculations show that the anisotropy energy decreases toward its bulk value as $\mathrm{Mn}$ is moved further away from the surface.

It is important to point out that the qualitative behavior of the magnetic anisotropy energy landscape (for the relevant case
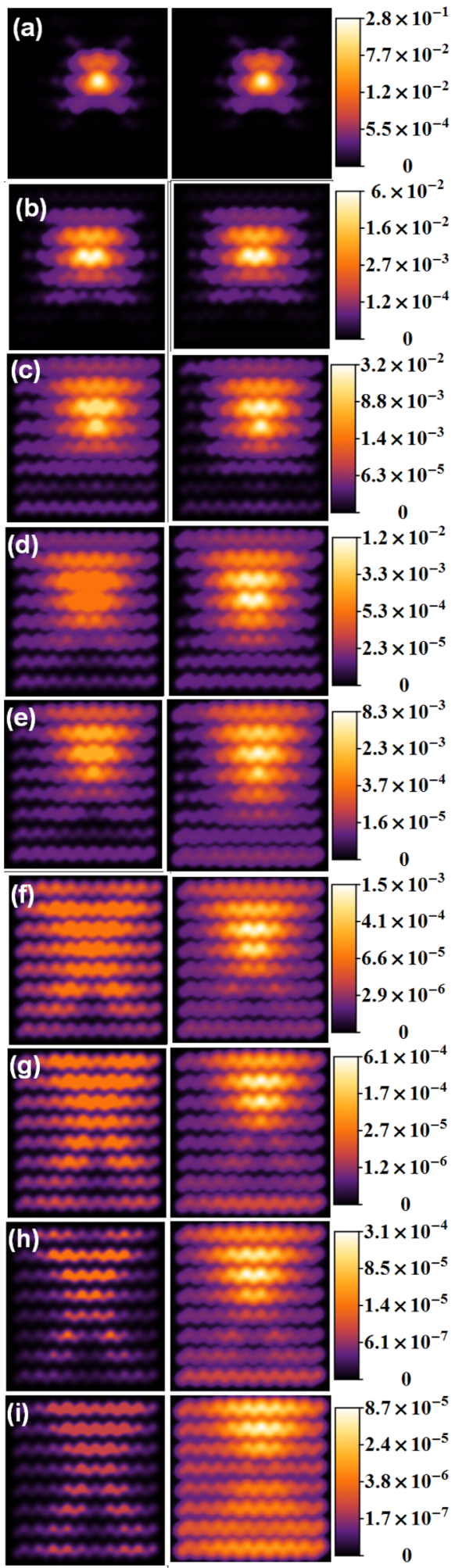

FIG. 10. (Color online) The (110) surface LDOS as a function of Mn depth. Panels (a) to (i) correspond to the LDOS calculated for Mn in sublayers 1 to 9 , respectively. The left and the right columns show the LDOS for easy and hard direction, respectively.

of a Mn close to the surface) remains unchanged with the size of the cluster. This includes the landscape of the anisotropy energy (e.g., easy and hard axes) as well as the anisotropy energy as a function of Mn depth (Fig. 11). Calculations carried 


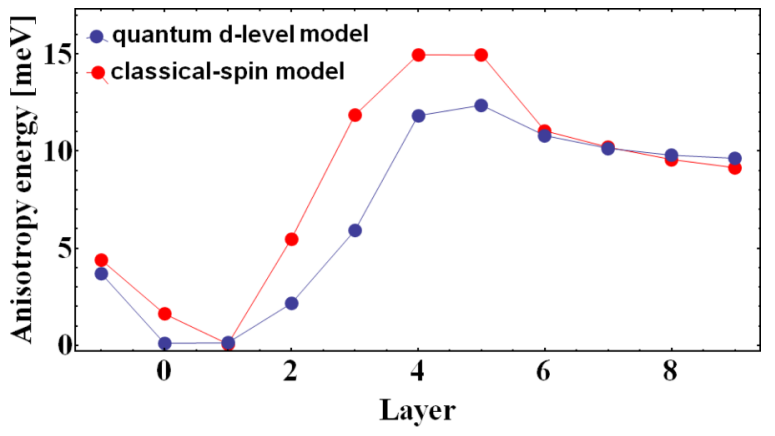

FIG. 11. (Color online) Magnetic anisotropy energy as a function of Mn depth. The values on the horizontal axis correspond to the sublayer index, in which the Mn impurity is located. The value at zero is the magnetic anisotropy energy of the (110) surface. The value before zero on the horizontal axis shows the magnetic anisotropy energy of the bulk. Red curve is for the case of the classical-spin model and blue curve shows the result obtained with the microscopic TB model, which includes the Mn $d$ levels (quantum $d$-level model).

out on much larger clusters [51] show that the qualitative behavior of magnetic anisotropy in Figs. 9 and 11 remains intact as a function of the cluster size, while the value of anisotropy energy saturates to a smaller value without any qualitative change.

Note that the initial increase of the anisotropy energy up to the fifth sublayer, and its subsequent decrease, also reported in Ref. [20], is most likely due to the quasidegeneracy between the last occupied and the acceptor states. It can be explained intuitively by looking at Fig. 10. The acceptor wave function becomes more extended as Mn moves away from the first sublayer. Such an extended wave function will be strongly affected by the surface until the Mn is moved deep enough so that the surface effects start to diminish (this corresponds to the sixth sublayer). A very small magnetic anisotropy of the first sublayer (of the order of $0.06 \mathrm{meV}$ ) is due to its highly localized wave function [see Fig. 10(a)]. The acceptor wave function in this case is less anisotropic, compared to the surface acceptor, which can be seen from the LDOS for easy and hard directions. The easy or hard direction here refers to a direction of the quantization axis for which the ground-state energy of the system is minimum or maximum, respectively.

The comparison between the anisotropy energy of Mn calculated with the two models seems to indicate that the difference is most pronounced on the surface and in the first five sublayers below the surface. As we mentioned earlier, this is due to the fact that the surface acceptor state in our fully quantum TB model is a deeper acceptor compared to its classical counterpart, which leads to a lower anisotropy energy. Another difference between the two models is illustrated in Fig. 12, where we plot the difference of the ground-state (GS) and the acceptor-level anisotropy energy, as a function of the Mn depth. For the classical-spin model it was found that the energy of the (single-particle) acceptor level $\epsilon_{\text {acc }}(\theta, \phi)$ and (many-particle) GS energy of the system $E(\theta, \phi)$ are very accurately related by

$$
\epsilon_{\mathrm{acc}}(\theta, \phi)=-E(\theta, \phi)+C,
$$

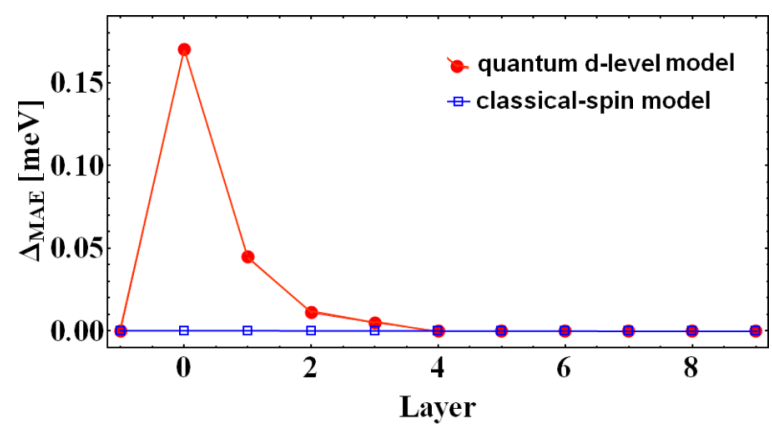

FIG. 12. (Color online) Difference $\Delta_{\mathrm{MAE}}$ between the groundstate and the acceptor magnetic anisotropy energy as a function of Mn depth. See Eq. (10). The notation on the horizontal axis is the same as in Fig. 11. The red line is the result for the quantum $d$-level model. The blue line $\left(\Delta_{\mathrm{MAE}}=0\right)$ is the result for the classical-spin model.

where $C$ is a constant independent of $\theta, \phi$. This means that the sum of the two energies $E(\theta, \phi)$ and $\epsilon_{\mathrm{acc}}(\theta, \phi)$ is the same for any direction of the Mn magnetic moment. If $\left(\theta_{\max }, \phi_{\max }\right)$ and $\left(\theta_{\min }, \phi_{\min }\right)$ define the two directions where $E(\theta, \phi)$ attains its maximum and minimum value respectively, from Eq. (8) we obtain

$$
\begin{aligned}
& {\left[E\left(\theta_{\max }, \phi_{\max }\right)-E\left(\theta_{\min }, \phi_{\min }\right)\right]} \\
& \quad+\left[\epsilon_{\mathrm{acc}}\left(\theta_{\max }, \phi_{\max }\right)-\epsilon_{\mathrm{acc}}\left(\theta_{\min }, \phi_{\min }\right)\right]=0 .
\end{aligned}
$$

The quantity $\left[E\left(\theta_{\max }, \phi_{\max }\right)-E\left(\theta_{\min }, \phi_{\min }\right)\right]$ is by definition the magnetic anisotropy of the system, MAE. Similarly, Eq. (8) implies that $\left[\epsilon_{\mathrm{acc}}\left(\theta_{\max }, \phi_{\max }\right)-\epsilon_{\mathrm{acc}}\left(\theta_{\min }, \phi_{\min }\right)\right]$ is the opposite of the magnetic anisotropy of the acceptor level, (-MAE) acc. Therefore, we can rewrite Eq. (9) as

$$
\Delta_{\mathrm{MAE}} \equiv \mathrm{MAE}-(\mathrm{MAE})_{\mathrm{acc}}=0 .
$$

Equations (8) and (10) are very useful and powerful. They imply that the total anisotropy of the system is essentially determined by the anisotropy of the single-particle acceptor level. This picture remains valid as long as the coupling to the conduction band is not sensitive to the magnetization orientation. It turns out that in the case of the quantum $d$-level TB model, Eq. (8) is not exactly satisfied. As a result, the quantity $\Delta_{\mathrm{MAE}}$ is not exactly zero, although, as shown in Fig. 12, its value is negligible for most of the cases, except for the surface and the first sublayers.

We suggest that the small change in the difference of the GS and the acceptor anisotropy energy is due to the inclusion of the $d$ orbitals, which brings about a magnetization-direction dependence coupling with the conduction band. In the classical-spin model, the majority $d$ electrons are essentially represented by a classical vector of fixed value $+5 / 2 \mu_{B}$, which only affects the (occupied) energy levels of the valence band through its SOI-induced orientation dependence. In contrast, our quantum $d$-level model includes the impurity $d$ orbitals and the hopping between the $d$ orbitals and the nearest-neighbor As atoms explicitly in the Hamiltonian. Unoccupied spin-down (minority) $d$ levels, located way up in the conduction band, hybridize with like-spin As $p$ orbitals of the valence band. This coupling is responsible for the small deviation from Eq. (10), 


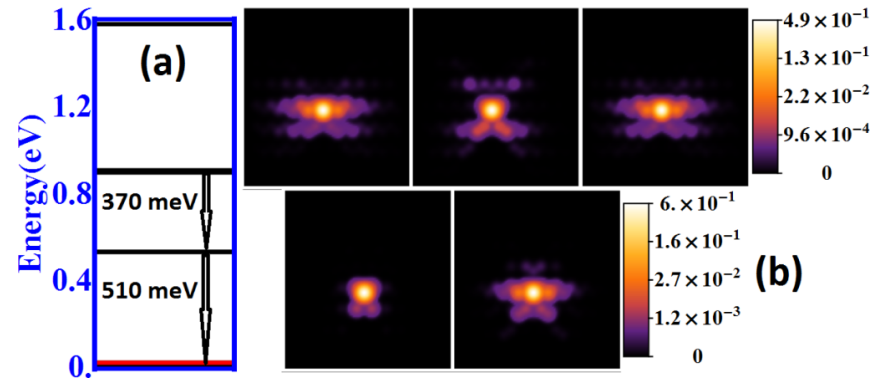

FIG. 13. (Color online) (a) Electronic structure of the Fe dopant in bulk GaAs showing the three $t_{2 g}$ and the two $e_{g}$ levels inside the gap. The red line indicates the Fermi level. (b) The (110) cross-sectional LDOS for the five $d$ levels in the gap. Top panels are for the $t_{2 g}$ triplet and the bottom panels are for the $e_{g}$ doublet.

which is also affected by the distance of the Mn atom from the surface. as shown in Fig. 12.

\section{B. Fe dopants on (110) GaAs surface}

As we showed in Sec. IV A, despite quantitative differences between the classical and the fully quantum treatments of the impurity magnetic moment, the classical spin model gives a good estimate of magnetic and electronic properties of the $\left[\mathrm{Mn}^{2+}, h\right]^{0}$ state, with a half-filled $3 d$ shell of $\mathrm{Mn}$ and a bound acceptor state. However, when the electronic transitions within the $d$-level shell of the dopant are important as in the case of $\mathrm{Fe}$ in $\mathrm{GaAs}$, the inclusion of $3 d$ electrons is necessary. In this section we present the results of our fully quantum microscopic TB model for Fe in the bulk and on the (110) surface of GaAs. Fe in the neutral acceptor state, $\left[\mathrm{Fe}^{2+}, h\right]^{0}$, in GaAs has six electrons in its $3 d$ shell plus a weakly bound hole. The transition from $\left[\mathrm{Fe}^{2+}, h\right]^{0}$ to the neutral isoelectronic state $\left[\mathrm{Fe}^{3+}\right]^{0}$ occurs when the minority $d$ electron occupies the hole bound to Fe atom. A fully unoccupied minority $d$ orbital in our TB represents the Fe atom in its isoelectronic state $\left(\left[\mathrm{Fe}^{3+}\right]^{0}\right)$, while the neutral acceptor state $\left(\left[\mathrm{Fe}^{2+}, h\right]^{0}\right)$ is realized when one electron from the valance band occupies one of the minority $d$ orbitals and creates an electron-hole excitation (see Fig. 1).

Figure 13 shows the electronic structure and the LDOS of a single Fe impurity in the bulk. The minority doublet $\left(e_{g}\right)$ level lies at $510 \mathrm{meV}$ above the top of the valence band while the minority triplet $\left(t_{2 g}\right)$ level is found at $370 \mathrm{meV}$ above the $e_{g}$ level. This bulk level structure is in agreement with the results reported previously [27,29]. The LDOS for each of the degenerate $e_{g}$ and $t_{2 g}$ levels are shown in the bottom and in the top of Fig. 13(b), respectively. The wave functions of the twofold degenerate level are highly localized and $60 \%$ of the spectral weight is located at the Fe site. In the case of the threefold degenerate level almost $50 \%$ of the spectral weight is located on the Fe atom. The lower symmetry of the surface compared to the bulk changes the electronic structure of the impurity. Figure 14(e) shows the energy level structure inside the gap for Fe positioned on the (110) GaAs surface. As one can clearly see from the figure, all of the levels inside the gap, which are primarily originating from the $\mathrm{Fe}$ $d$ orbitals, are split. The two unoccupied levels immediately

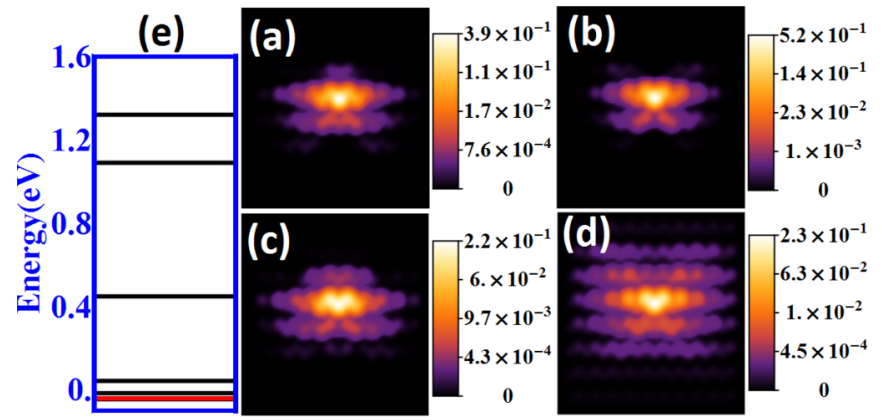

FIG. 14. (Color online) Electronic structure and LDOS for the Fe dopant on the (110) GaAs surface. (a)-(d) The (110) cross-sectional LDOS for the four topmost levels in the gap, with (a) being the level with the highest energy and (d) the level with the lowest energy. (e) Electronic level structure showing the symmetry-broken $t_{2 g}$ and $e_{g}$ levels inside the gap. The red line indicates the Fermi level.

above the Fermi level are closer to the valence band edge and are therefore more delocalized around the impurity. The splitting between these two levels is $\approx 60 \mathrm{meV}$. The LDOS calculated for this pair of states is plotted in Fig. 14(d). The other three levels in the gap appear at $0.45 \mathrm{eV}, 1.1 \mathrm{eV}$, and $1.3 \mathrm{eV}$. The corresponding LDOS images for each of the levels are plotted in Figs. 14(a)-14(c). These distinct energy states appear to be more localized on the impurity compared to the states which are closer to the valence band edge. The shape of these acceptor states bears a striking resemblance to the STM topographies of $\mathrm{Fe}$ impurities on the GaAs surface, reported by Richardella et al. (see Fig. 2(c) in Ref. [26]). However, we find differences in the exact positions of the levels in the gap, compared to the experimental data. In fact, Richardella et al. found two Fe-induced peaks in their spectroscopic data, located at $0.87 \pm 0.05 \mathrm{eV}$ and $1.52 \pm 0.05 \mathrm{eV}$, respectively. We note, however, that due to the finite energy resolution of the STM, the existence of more than one energy level within the width of a broad peak in the experimental spectroscopic data is not unlikely. In addition, the positions of the peaks can be modified by the tip-induced band bending. One should also take into account that the splitting between the energy levels in the gap are sensitive to the amount of the lattice distortion.

The above considerations may provide an explanation for the quantitative differences in the in-gap level structure obtained with our TB model and that reported in Ref. [26]. Finally, we note that in a more recent experimental study [28], the authors found evidence of six peaks in their $d I / d V$ measurements for Fe on the (110) GaAs surface. In particular, one of the observed peaks can be related to the two closely spaced energy levels in the vicinity of the Fermi energy that we found in our TB calculations (see Fig. 14). The occupancy of the levels close to the Fermi energy can be different depending on the valence state of Fe, namely $\left[\mathrm{Fe}^{2+}, h\right]^{0}$ and $\left[\mathrm{Fe}^{3+}\right]^{0}$.

Figure 15 shows the magnetic anisotropy energy of the $\mathrm{Fe}$ impurity in the $\left[\mathrm{Fe}^{3+}\right]^{0}$ state for different directions of the quantization axis. The surface anisotropy energy is approximately $1 \mathrm{meV}$, while Fe in bulk GaAs displays a considerably smaller anisotropy energy, of the order of $10^{-4} \mathrm{meV}$. The 

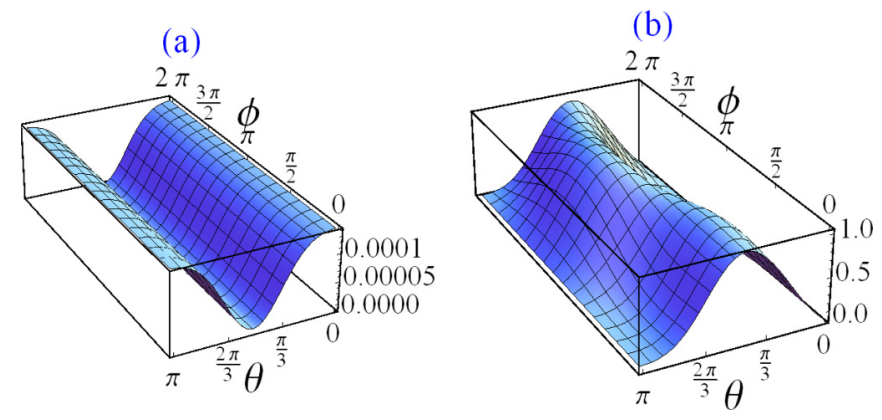

FIG. 15. (Color online) Magnetic anisotropy landscape for the Fe dopant in bulk GaAs (a) and Fe on the (110) GaAs surface (b). Energy is in units of meV.

magnetic anisotropy energy landscape for the $\left[\mathrm{Fe}^{2+}, h\right]^{0}$ state (not shown here) is qualitatively and quantitatively similar to that of $\left[\mathrm{Mn}^{2+}, h\right]^{0}$.

In Fig. 16 we compare the anisotropy energy for the two valence states of $\mathrm{Fe},\left[\mathrm{Fe}^{3+}\right]^{0}$ and $\left[\mathrm{Fe}^{2+}, h\right]^{0}$, as the impurity atom is moved down from the (110) surface toward the center of the cluster. The qualitative and quantitative difference between the anisotropy energy in the two valence states is remarkable. While for $\left[\mathrm{Fe}^{2+}, h\right]^{0}$ the sublayer dependence shows strong similarities with the case of $\left[\mathrm{Mn}^{2+}, h\right]^{0}$, the anisotropy for the the isoelectronic $\left[\mathrm{Fe}^{3+}\right]^{0}$ state is quite different: it is typically one order of magnitude smaller that for $\left[\mathrm{Fe}^{2+}, h\right]^{0}$ and depends weakly on the sublayer. This striking behavior can be easily understood on the basis of the discussion leading to Eqs. (8)-(10). As for the Mn impurity, the total magnetic anisotropy of the system is closely related to the magnetic anisotropy of the acceptor levels. As seen in Fig. 1, in the isoelectronic $\left[\mathrm{Fe}^{3+}\right]^{0}$ state this $p$-orbital-like acceptor level is occupied by the extra electron that Fe has with respect to $\mathrm{Mn}$. Therefore the total energy of the system contains also the contribution of this level. Then Eq. (10) implies that most of the anisotropy coming from all the other occupied levels of the valance band is essentially canceled by the approximately equal and opposite contribution coming from the occupied acceptor. In contrast, in the $\left[\mathrm{Fe}^{2+}, h\right]^{0}$ valence state, the extra electron occupies one of the higher minority $d$-orbital levels in the gap, and the acceptor level is empty (or is occupied by

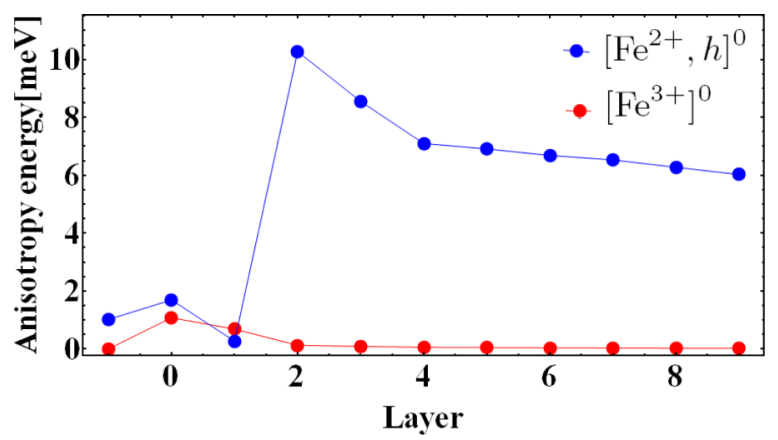

FIG. 16. (Color online) Magnetic anisotropy energy as a function of the Fe depth. The notations on the horizontal are the same as in Fig. 11, with zero corresponding to the surface layer and the value below zero to the bulk result. Red dots are for the $\left[\mathrm{Fe}^{3+}\right]^{0}$ state and blue dots for the $\left[\mathrm{Fe}^{2+}, h\right]^{0}$ state. a "hole"). Thus, as for the $\left[\mathrm{Mn}^{2+}, h\right]^{0}$, the cancellation does not occur. Furthermore, the anisotropy of the topmost $d$-orbital level is, as expected, small. Therefore the behavior of $\left[\mathrm{Fe}^{2+}, h\right]^{0}$
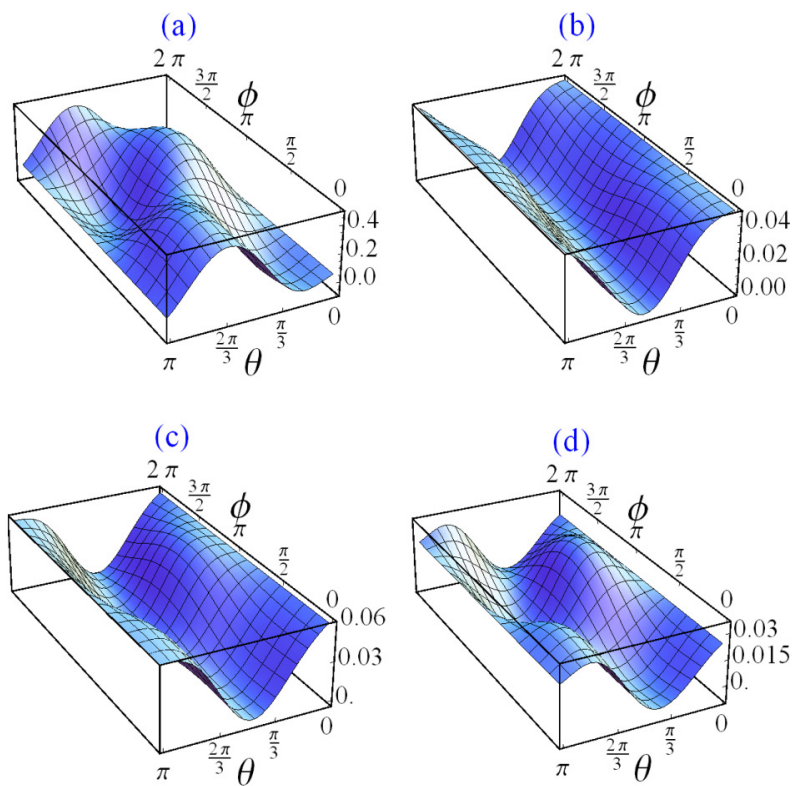

(e)
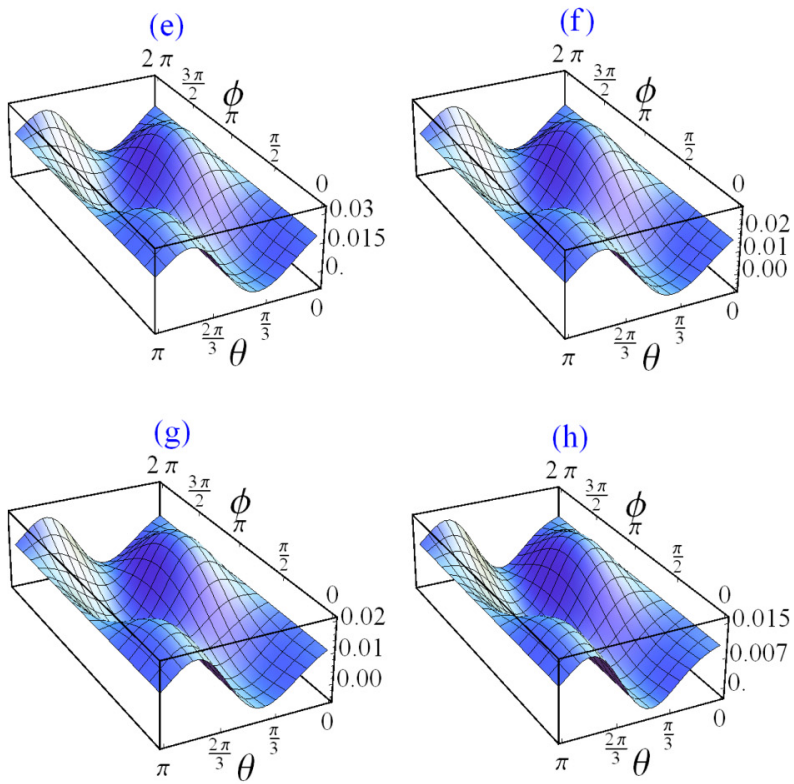

(i)

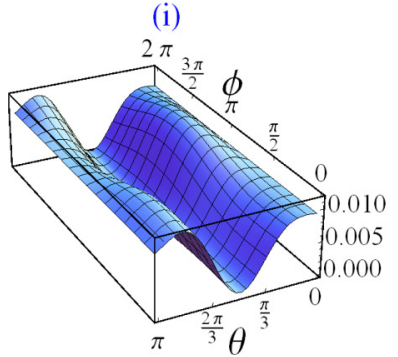

FIG. 17. (Color online) Magnetic anisotropy energy landscapes for $\mathrm{Fe}$ in the $\left[\mathrm{Fe}^{3+}\right]^{0}$ electronic configuration as a function of the impurity position below the surface. Panels (a) to (i) correspond to $\mathrm{Fe}$ in the first to ninth sublayer, respectively. Energy is in units of meV. 
is quite similar to $\left[\mathrm{Mn}^{2+}, h\right]^{0}$. These simple considerations lead us to predict that, at least within our non-self-consistent treatment, the charged states $\left[\mathrm{Fe}^{2+}\right]^{-}$and $\left[\mathrm{Fe}^{3+}, h\right]^{+}$should have the same behavior of $\left[\mathrm{Fe}^{3+}\right]^{0}$ and $\left[\mathrm{Fe}^{2+}, h\right]^{0}$ respectively, since the occupancy of the acceptor state is the same. This is exactly what our calculations show. Again, we emphasize that our non-self-consistent calculations should be taken cautiously when charged states are involved.
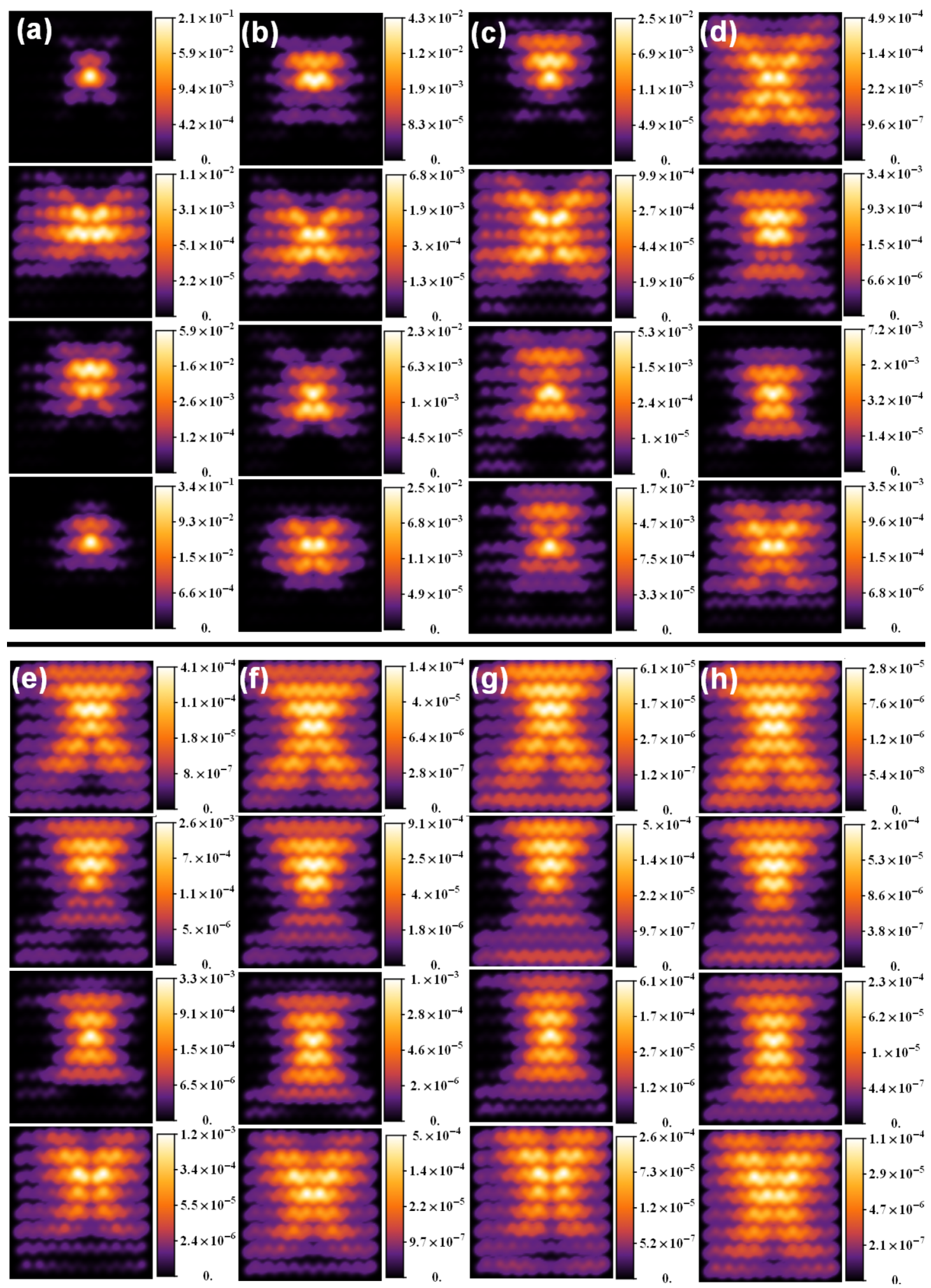

FIG. 18. (Color online) The (110) surface LDOS for four Fe-induced levels in the gap as a function of the Fe depth. Panels (a) to (h) correspond to $\mathrm{Fe}$ in the first to eighth sublayer, respectively. 
The importance of this result stems from the fact that the valence and charge state of individual TM impurities in GaAs can presently be manipulated with a variety of techniques. For example, as mentioned earlier, it is possible to switch the impurity from the $\left[\mathrm{Fe}^{3+}\right]^{0}$ to the $\left[\mathrm{Fe}^{2+}\right]^{-}$ state via a voltage-dependent local manipulation of the Fermi level by means of tip-induced band bending in STM experiments [27]. A similar manipulation of the Fermi level might soon allow the switching between the $\left[\mathrm{Fe}^{3+}\right]^{0}$ and the $\left[\mathrm{Fe}^{3+}, h\right]^{+}$charge state. On the other hand, optical manipulation of the valence state might permit switching between the $\left[\mathrm{Fe}^{3+}\right]^{0}$ and the $\left[\mathrm{Fe}^{2+}, h\right]^{0}$ states. Our calculations indicate that the switching between these valence and charged states should be also accompanied and characterized by significant changes in the magnetic anisotropy energy of the system.

We would like to mention that for an Fe impurity in bulk, our ab initio calculations, in agreement with previously published results, show that the splitting of the (minority) $d$ levels in the GaAs gap follows the behavior described in Fig. 1. This figure shows that the dominant effect of the splitting comes from the tetragonal symmetry of the lattice. Effects coming from the spin-orbit interaction, which are certainly present for the $d(l=2)$ minority-spin level occupied by the sixth electron in $\mathrm{Fe}$, seem to be small. The situation for the Fe dopant on the surface is more complex since, as we have already described, in this case the surface-induced broken symmetry contributes substantially to the splitting, in a way that is not easily disentangled from other, more intrinsic, mechanisms. In any case, it is quite remarkable that the behavior of the anisotropy for the $\left[\mathrm{Fe}^{2+}, h\right]^{0}$ complex resembles qualitatively the behavior of the $\left[\mathrm{Mn}^{2+}, h\right]^{0}$ : in both cases the total anisotropy is controlled by the anisotropy of the impurity acceptor level, regardless of whether or not an extra electron is present.

Figure 17 shows the magnetic anisotropy landscape for the Fe impurity in the isoelectronic state as a function of its position below the surface. Although the results for the surface layer [Fig. 15(b)] and for the first sublayer [Fig. 16(a)] are similar, in the latter case the magnetic anisotropy energy is smaller. As the Fe atom is moved away from the surface, the behavior of the magnetic anisotropy approaches that found in the bulk. When Fe is placed in the ninth sublayer, i.e., in the middle of the cluster, we expect it to behave like Fe in the bulk. This is confirmed in Fig. 17(i), which shows that the anisotropy energy has now decreased down to $10^{-2} \mathrm{meV}$ compared to $1 \mathrm{meV}$ for the surface and the anisotropy landscape is almost identical to its bulk counterpart [see Fig. 15(a)]. Figure 18 shows the LDOS for the four top-most Fe-induced levels in the gap when the spin quantization axis is along the easy direction. As the $\mathrm{Fe}$ atom is moved down from the surface towards the center of the cluster, the concentration of the spectral weight on the impurity site decreases and the LDOS becomes more delocalized. The butterfly shape of the LDOS around the impurity is more pronounced for the sublayers located farther away from the surface. The surface LDOS for $\mathrm{Fe}$ in the sublayers close to the surface is not very sensitive to the direction of the spin quantization axis. However, we find that when Fe is placed between the fifth and the eighth sublayer, the calculated LDOS images do
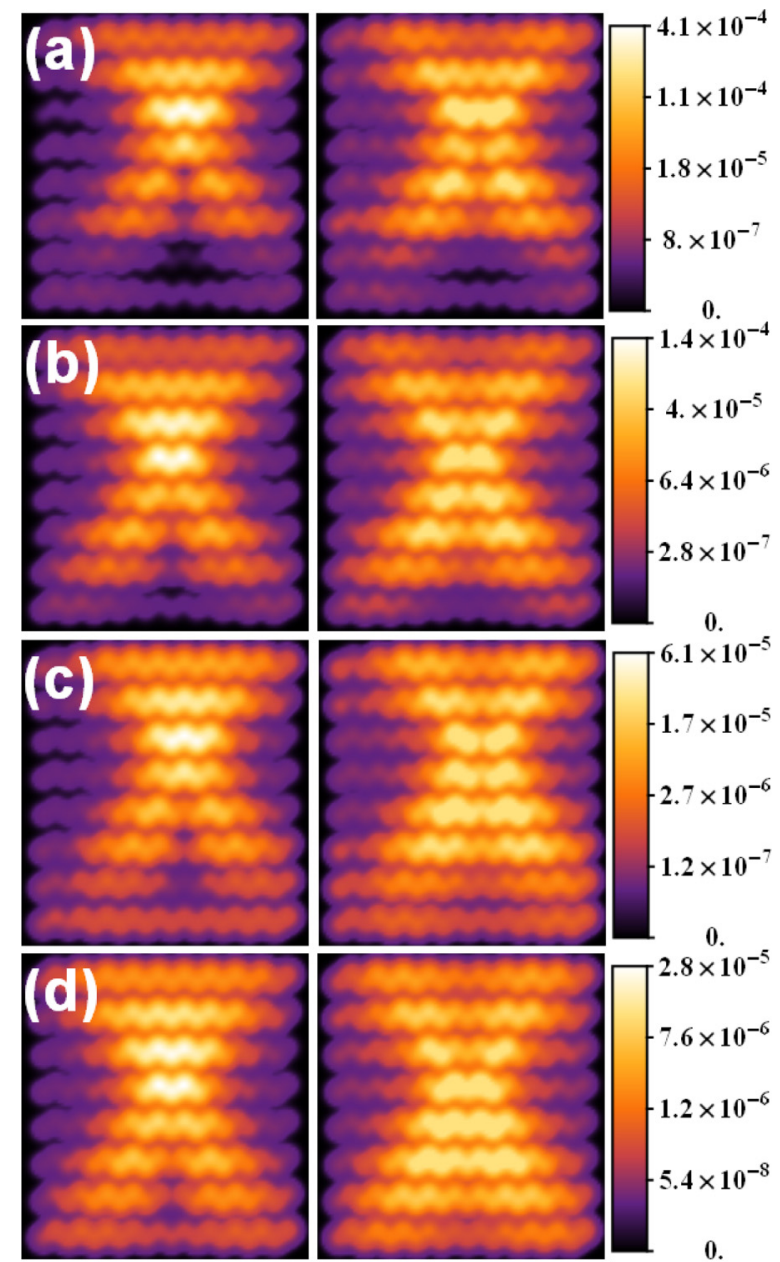

FIG. 19. (Color online) The (110) surface LDOS for the topmost level in the gap for two different directions of the quantization axis. Panels (a) to (d) correspond to $\mathrm{Fe}$ in the fifth to eighth sublayer, respectively. The left (right) column shows the LDOS for the quantization axis along the easy (hard) direction.

display visible differences for the easy and hard directions, as shown in Fig. 19. These changes might well be detectable by STM, when the direction of the impurity magnetic moment is changed by an external magnetic field. An estimation based on the maximum anisotropy energy of $0.05 \mathrm{meV}$ (for the fifth sublayer which decreases to $0.026 \mathrm{meV}$ for the eighth sublayer) for the Fe on its isoelectronic state, suggests that it should be possible to manipulate the spin of the impurity with magnetic fields of the order of $10^{-1} \mathrm{~T}$. This is very different from the case of Mn on the (110) GaAs surface. As was demonstrated in Ref. [24] both theoretically and experimentally, due to the strongly localized character of the Mn acceptor wave function on the surface and the large magnetic anisotropy energy of $\mathrm{Mn}$ in the near-surface layers, the acceptor hole LDOS is practically insensitive to the direction of the Mn magnetic moment in magnetic fields up to $6 \mathrm{~T}$. In the case of $\mathrm{Fe}$ in its isoelectronic state, the combination of lowanisotropy energy and the sensitivity of the surface LDOS to the direction of the impurity magnetic moment makes such manipulation possible with magnetic fields well within the experimental range. 


\section{CONCLUSIONS}

In this paper we have studied the electronic structure and magnetic properties of individual $\mathrm{Mn}$ and $\mathrm{Fe}$ dopants in the bulk and near the (110) surface of GaAs. Our theoretical treatment is based on a microscopic TB model including explicitly the $d$ orbitals of the dopant. We have employed DFT calculations to obtain the spin-resolved density of states for the impurity $d$ states, which was then used to determine the TB parameters for the $d$ orbitals. We calculated the in-gap electronic level-structure, LDOS, and magnetic anisotropy landscapes for Mn and Fe impurities positioned on the surface or in subsurface layers.

Our calculations for Mn are typically in good agreement with the results obtained by a TB model where the impurity magnetic moment is treated as an effective classical spin. In particular, we reproduced the well-known features of $\mathrm{Mn}$ in GaAs, such as the position of the acceptor level in the gap and the spatial character of its wave function both in bulk and on the surface, which are also in agreement with experimental results. However, the microscopic quantum model finds a lower magnetic anisotropy energy for a Mn dopant on the surface compared to the classical-spin model. The difference between the anisotropy energy in the two models stems from the deeper and therefore the considerably more localized character of the acceptor state on the surface, found in the quantum $d$-level model.

For the case of $\mathrm{Fe}$ in bulk, the microscopic model correctly finds two degenerate minority-spin levels in the gap, of predominately $d$ character, with the expected twofold and threefold degeneracy associated with the $e_{g}$ and $t_{2 g}$ symmetry, respectively. The structure of these minority $d$-character levels changes significantly when Fe is placed on the surface or in the nearby sublayers. Indeed, we find that the orbital degeneracy is lifted by surface effects, and the electronic structure consists of five unoccupied nondegenerate levels in the gap, two of which are very close to the top of valence band, two in the middle, and one close to the conduction band. We were able to make connections between the calculated in-gap level structure and the experimental spectroscopic data for $\mathrm{Fe}$ on the (110) GaAs surface.
We also presented the calculated anisotropy energy landscapes and the LDOS of the in-gap states for Fe near the (110) surface. Importantly, we found that the anisotropy energy of the Fe impurity on the surface and subsurfaces depends on its valence state. Although the neutral acceptor state $\left[\mathrm{Fe}^{2+}, h\right]^{0}$ behaves similarly to $\mathrm{Mn}$, for the isoelectronic state $\left[\mathrm{Fe}^{3+}\right]^{0}$ the anisotropy energy is considerably smaller, and behaves differently as a function of the impurity depth and the orientation of its magnetic moment. For an $\mathrm{Fe}$ dopant positioned in between the fifth to eighth sublayers, the anisotropy energy drops to a fraction of a meV. At the same time the spatial profile of the wave function associated with the topmost impurity level in the gap is considerably extended and therefore sensitive to the orientation of the impurity magnetic moment. This situation is quite different from the case of the Mn acceptor [24], whose wave function is strongly localized for a dopant on the surface (where the anisotropy is small) and extended for a dopant in the sublayers (where, however, the anisotropy is large). These special features of the Fe dopant will permit the manipulation of the Fe spin by means of an external magnetic field of few teslas. Under this occurrence, the results of our calculations predict that, for an Fe dopant in its isoelectronic state, positioned a few monolayers below the (110) GaAs surface, a change in the direction of the dopant magnetic moment will produce a detectable difference in the STM cross-sectional view of the LDOS of the impurity levels in the gap.

\section{ACKNOWLEDGMENTS}

It is a pleasure to thank A. H. MacDonald, M. E. Flatté, and P. M. Koenraad for very useful discussions and collaboration. This work was supported by the Faculty of Technology at Linnaeus University, by the Swedish Research Council under Grant No. 621-2010-3761, and the NordForsk research network 080134 "Nanospintronics: Theory and Simulations." Computational resources have been provided by the Lunarc center for scientific and technical computing at Lund University.
[1] P. M. Koenraad and M. E. Flatté, Nat. Mater. 10, 91 (2011).

[2] A. M. Yakunin, A. Y. Silov, P. M. Koenraad, J. H. Wolter, W. Van Roy, J. De Boeck, J.-M. Tang, and M. E. Flatté, Phys. Rev. Lett. 92, 216806 (2004).

[3] T. Shinada, S. Okamoto, T. Kobayashi, and I. Ohdomari, Nature (London) 437, 1128 (2005).

[4] D. Kitchen, A. Richardella, J.-M. Tang, M. E. Flatté, and A. Yazdani, Nature (London) 442, 436 (2006).

[5] F. Marczinowski, J. Wiebe, J.-M. Tang, M. E. Flatté, F. Meier, M. Morgenstern, and R. Wiesendanger, Phys. Rev. Lett. 99, 157202 (2007).

[6] D. H. Lee and J. A. Gupta, Science 330, 1807 (2010).

[7] J. K. Garleff, A. P. Wijnheijmer, A. Y. Silov, J. van Bree, W. Van Roy, J.-M. Tang, M. E. Flatté, and P. M. Koenraad, Phys. Rev. B 82, 035303 (2010).
[8] M. Fuechsle, J. A. Miwa, S. Mahapatra, H. Ryu, S. Lee, O. Warschkow, L. C. L. Hollenberg, G. Klimeck, and M. Y. Simmons, Nat. Nanotechnol. 7, 242 (2012).

[9] J. J. Pla, K. Y. Tan, J. P. Dehollain, W. H. Lim, J. Morton, D. N. Jamieson, A. S. Dzurak, and A. Morello, Nature (London) 489, 541 (2012).

[10] Y. Zhao, P. Mahadevan, and A. Zunger, Appl. Phys. Lett. 84, 3753 (2004).

[11] P. Mahadevan, A. Zunger, and D. D. Sarma, Phys. Rev. Lett. 93, 177201 (2004).

[12] A. Mikkelsen, B. Sanyal, J. Sadowski, L. Ouattara, J. Kanski, S. Mirbt, O. Eriksson, and E. Lundgren, Phys. Rev. B 70, 085411 (2004).

[13] A. Stroppa, X. Duan, M. Peressi, D. Furlanetto, and S. Modesti, Phys. Rev. B 75, 195335 (2007). 
[14] H. Ebert and S. Mankovsky, Phys. Rev. B 79, 045209 (2009).

[15] M. F. Islam and C. M. Canali, Phys. Rev. B 85, 155306 (2012).

[16] J.-M. Tang and M. E. Flatté, Phys. Rev. Lett. 92, 047201 (2004).

[17] J.-M. Tang and M. E. Flatté, Phys. Rev. B 72, 161315 (2005).

[18] C. Timm and A. H. MacDonald, Phys. Rev. B 71, 155206 (2005).

[19] J.-M. Jancu, J.-C. Girard, M. O. Nestoklon, A. Lemaitre, F. Glas, Z. Z. Wang, and P. Voisin, Phys. Rev. Lett. 101, 196801 (2008).

[20] T. O. Strandberg, C. M. Canali, and A. H. MacDonald, Phys. Rev. B 80, 024425 (2009).

[21] T. O. Strandberg, C. M. Canali, and A. H. MacDonald, Phys. Rev. B 81, 054401 (2010).

[22] T. O. Strandberg, C. M. Canali, and A. H. MacDonald, Phys. Rev. Lett. 106, 017202 (2011).

[23] J. Mašek, F. Máca, J. Kudrnovský, O. Makarovsky, L. Eaves, R. P. Campion, K. W. Edmonds, A. W. Rushforth, C. T. Foxon, B. L. Gallagher, V. Novák, J. Sinova, and T. Jungwirth, Phys. Rev. Lett. 105, 227202 (2010).

[24] M. Bozkurt, M. R. Mahani, P. Studer, J.-M. Tang, S. R. Schofield, N. J. Curson, M. E. Flatté, A. Y. Silov, C. F. Hirjibehedin, C. M. Canali, and P. M. Koenraad, Phys. Rev. B 88, 205203 (2013).

[25] J. K. Garleff, C. Çelebi, W. Van Roy, J.-M. Tang, M. E. Flatté, and P. M. Koenraad, Phys. Rev. B 78, 075313 (2008).

[26] A. Richardella, D. Kitchen, and A. Yazdani, Phys. Rev. B 80, 045318 (2009).

[27] J. Bocquel, V. R. Kortan, C. Sahin, R. P. Campion, B. L. Gallagher, M. E. Flatté, and P. M. Koenraad, Phys. Rev. B 87, 075421 (2013).

[28] S. Mühlenberend, M. Gruyters, and R. Berndt, Phys. Rev. B 88, 115301 (2013).

[29] E. Malguth, A. Hoffmann, and M. R. Phillips, Phys. Status Solidi B 245, 455 (2008).

[30] D. J. Chadi, Phys. Rev. B 16, 790 (1977).

[31] J. C. Slater and G. F. Koster, Phys. Rev. 94, 1498 (1954).

[32] D. A. Papaconstantopoulos and M. J. Mehl, J. Phys.: Condens. Matter 15, R413 (2003).

[33] J.-M. Jancu, R. Scholz, F. Beltram, and F. Bassani, Phys. Rev. B 57, 6493 (1998).
[34] D. J. Chadi, Phys. Rev. Lett. 41, 1062 (1978).

[35] D. J. Chadi, Phys. Rev. B 19, 2074 (1979).

[36] C. Çelebi, J. K. Garleff, A. Y. Silov, A. M. Yakunin, P. M. Koenraad, W. Van Roy, J.-M. Tang, and M. E. Flatté, Phys. Rev. Lett. 104, 086404 (2010).

[37] A. M. Yakunin, A. Y. Silov, P. M. Koenraad, J.-M. Tang, M. E. Flatté, J.-L. Primus, W. V. Roy, J. D. Boeck, A. M. Monakhov, K. S. Romanov, I. E. Panaiotti, and N. S. Averkiev, Nat. Mater. 6, 512 (2007).

[38] Y. M. Niquet, D. Rideau, C. Tavernier, H. Jaouen, and X. Blase, Phys. Rev. B 79, 245201 (2009).

[39] M. Zieliński, Phys. Rev. B 86, 115424 (2012).

[40] P. Blaha, K. Schwarz, G. K. H. Madsen, D. Kvasnicka, and J. Luitz, WIEN2k, An Augmented Plane Wave Plus Local Orbitals Program for Calculating Crystal Properties (Vienna University of Technology, Vienna, 2001).

[41] J. P. Perdew, K. Burke, and M. Ernzerhof, Phys. Rev. Lett. 77, 3865 (1996).

[42] J. M. Soler, E. Artacho, J. D. Gale, A. García, J. Junquera, P. Ordejón, and D. Sánchez-Portal, J. Phys.: Condens. Matter 14, 2745 (2002).

[43] V. I. Anisimov, J. Zaanen, and O. K. Andersen, Phys. Rev. B 44, 943 (1991).

[44] K. Sato, L. Bergqvist, J. Kudrnovský, P. H. Dederichs, O. Eriksson, I. Turek, B. Sanyal, G. Bouzerar, H. KatayamaYoshida, V. A. Dinh, T. Fukushima, H. Kizaki, and R. Zeller, Rev. Mod. Phys. 82, 1633 (2010).

[45] B. Belhadji, L. Bergqvist, R. Zeller, P. H. Dederichs, K. Sato, and H. Katayama-Yoshida, J. Phys.: Condens. Matter 19, 436227 (2007).

[46] P. Gopal and N. A. Spaldin, Phys. Rev. B 74, 094418 (2006).

[47] W. Schairer and M. Schmidt, Phys. Rev. B 10, 2501 (1974).

[48] T. Lee and W. W. Anderson, Solid State Commun. 2, 265 (1964).

[49] R. A. Chapman and W. G. Hutchinson, Phys. Rev. Lett. 18, 443 (1967).

[50] M. Linnarsson, E. Janzen, B. Monemar, M. Kleverman, and A. Thilderkvist, Phys. Rev. B 55, 6938 (1997).

[51] M. R. Mahani, A. Pertsova, and C. M. Canali, arXiv:1402.3069. 